\title{
Chemistry at surfaces: from ab initio structures to quantum dynamics
}

\author{
Giorgio Lanzani · Rocco Martinazzo • \\ Giuliana Materzanini • Ilaria Pino • \\ Gian Franco Tantardini
}

Received: 20 September 2006 / Accepted: 13 October 2006 / Published online: 5 January 2007

(C) Springer-Verlag 2006

\begin{abstract}
Recent years have witnessed an ever growing interest in theoretically studying chemical processes at surfaces. Apart from the interest in catalysis, electrochemistry, hydrogen economy, green chemistry, atmospheric and interstellar chemistry, theoretical understanding of the molecule-surface chemical bonding and of the microscopic dynamics of adsorption and reaction of adsorbates are of fundamental importance for modeling known processes, understanding new experimental data, predicting new phenomena, controlling reaction pathways. In this work, we review the efforts we have made in the last few years in this exciting field. We first consider the energetics and the structural properties of some adsorbates on metal surfaces, as deduced by converged, first-principles, plane-wave calculations within the slab-supercell approach. These studies comprise water adsorption on $\mathrm{Ru}(0001)$, a subject of very intense debate in the past few years, and oxygen adsorption on aluminum, the prototypical example of metal passivation. Next, we address dynamical processes at surfaces with classical and quantum methods. Here the main interest is in hydrogen dynamics on metallic and semi-metallic surfaces, because of its importance for hydrogen storage and interstellar chem-
\end{abstract}

G. Lanzani · R. Martinazzo (凶) . G. Materzanini · I. Pino ·

G. F. Tantardini

Department of Physical Chemistry and Electrochemistry,

University of Milan, V. Golgi 19, 20133 Milan, Italy

e-mail: rocco.martinazzo@unimi.it

R. Martinazzo · G. F. Tantardini

CIMAINA, University of Milan, V. Golgi 19, 20133 Milan, Italy

G. F. Tantardini

IMST, CNR, Institute of Molecular Science and Technology,

V. Golgi 19, 20133 Milan, Italy

e-mail: gianfranco.tantardini@unimi.it istry. Hydrogen sticking is studied with classical and quasi-classical means, with particular emphasis on the relaxation of hot-atoms following dissociative chemisorption. Hot atoms dynamics on metal surfaces is investigated in the reverse, hydrogen recombination process and compared to Eley-Rideal dynamics. Finally, Eley-Rideal, collision-induced desorption, and adsorbate-induced trapping are studied quantum mechanically on a graphite surface, and unexpected quantum effects are observed.

Keywords Molecule surface interactions . Adsorption and reaction at surfaces - Dynamics at surfaces · Dynamical simulations · Supercell DFT calculations

\section{Introduction}

Surfaces represent the borders of phases of matter and exhibit unusual chemical and physical properties. Surface atoms are undercoordinated compared to atoms in the bulk, and therefore chemically different from them. For example, substantial surface reconstruction may occur when cutting solid materials, giving rise to singular geometric arrangements in the very first layers; some metals do not mix in the bulk because of unfavorable energetics but form surface alloys, thanks to the unique environment at surfaces. This very unique environment makes surfaces so special and so important in a wide variety of fields, with deep impact both on economy and on fundamental science. Heterogeneous catalysis is involved in about one-third of the modern economy; (industrially relevant) electrochemical processes employ charged surfaces and polarized interfaces; surfaces are the favorite media for biological evolution, 
and biological systems improve by ever increasing their interface-to-volume ratio (e.g. the brain); hydrogen is the most abundant element in the universe, and molecular hydrogen formation in interstellar space occurs on the surface of the so-called cosmic dust, and profoundly affects the chemical and physical evolution of interstellar clouds. Surface science is just in between a number of disciplines; it benefits from and triggers the developments of these fields. It is a very active area of research which catalyzes the transfer of knowledge between different disciplines.

Theoretical surface science occupies a special position in this field. Because of its attitude in explaining surface processes at the atomic- scale resolution, it is of valuable help in rationalizing experimental data, and with the ever increasing availability and reliability of predicting theories, models, software tools and computational power, its importance is being recognized even by the more skeptical ones. "Theoretical design" of a catalyst has been recently accomplished on a world-wide important catalytic reaction (steam reforming) [1], valuable theoretical advice has been given for optimal catalysts in ammonia synthesis [2], and advances have been made in the in Silico dream, the chemistry lab in a computer, with first-principle determination of reaction rates at surfaces [3-5]. At the same time, continuous progresses in experimental techniques give rise to new, unexpected results and pose new challenges to theory. Very recently, for example, the long sought objective of achieving modeselective chemistry in a many-atom system [6] has been accomplished in surface chemistry: Liu et al. selectively desorbed hydrogen molecules from a mixed H/D covered $\mathrm{Si}(111)$ surface by using IR pulses resonant to the $\mathrm{H}-\mathrm{Si}$ stretching mode [7], but they were unable to provide a molecular mechanism for the process. A comprehensive outlook of modern surface science can be found in the book of Somorjai [8] and in a recent Surface Science special issue, in particular [9-16]. Groß' book [17] is a very well written book in theoretical surface science.

In this paper, we give an overview of our activity in this exciting research field. We first consider in Sect. 2 two examples of how first-principles methods allow to gain structural and energetic informations about adsorbed species at a surface. In Sect. 2.1 we consider water adsorption on a metal surface, the $\mathrm{Ru}(0001)$ surface, a topic of very intense debate in the past few years, after Feibelman's observation [18] that the experimentally derived geometric structure [19] of a water bilayer on $\mathrm{Ru}(0001)$ can only be explained by water dissociation upon adsorption. The second, structural problem we address in Sect. 2.2 is oxygen adsorption on an aluminum surface, a prototypical example to study metal passivation.
In Sect. 3 and 4 we consider dynamical processes at surfaces, studied by classical and quantum means. Here, we could not follow a systematic, first-principles approach, that is: firstly, determine structures and energetics of reagents, products and intermediates; secondly, compute an interaction potential for the active degrees of freedom and thirdly, follow the dynamics. We rather followed a pragmatic approach and used previously developed interaction potentials. In Sect. 3, we consider the $\mathrm{H}$-on-Ni system as a typical $\mathrm{H}$-on-metal system for which an accurate, semiempirical embedded-diatomicsin-molecule (EDIM) potential model was developed by Truhlar and coworkers [20,21]. The main advantage of such potential model, when compared to present-day first-principle potentials for hydrogen on metal surfaces, is the reliable description of lattice potential. The latter is essential to address important issues like energy relaxation phenomena, and cannot be neglected in studying, e.g., atom sticking. We used this potential model to study hot atom dynamics in two different cases. We first consider in Sect. 3.1 hot atom formation when an atomic hydrogen beam impinges on a H-covered surface, and compare hot atom dynamics with Eley-Rideal dynamics [22], i.e. direct molecular hydrogen formation. Then, in Sect. 3.2 we present some results of our ongoing research on hot atom dynamics following dissociative chemisorption of hydrogen molecules on the same surface, the $\mathrm{Ni}(100)$ surface.

In Sect. 4 we consider some aspects of hydrogen dynamics on graphite, an important issue for understanding formation of hydrogen molecules in the interstellar medium (ISM). In this case we used the previously derived density functional theory (DFT) potential energy surface of Jackson and coworkers [23,24] in a quantum study of collision induced processes involving hydrogen atoms. In Sect. 4.1 we consider the case in which the target hydrogen atom is chemisorbed on graphite, focusing on the quantum effects we found in Eley-Rideal and collision induced desorption (CID) cross-sections [25,26]. In Sect. 4.2 we consider the case in which the target atom is physisorbed on graphite, with some emphasis on the efficient projectile trapping due to scattering with the adsorbate.

We conclude in Sect. 5 with an outlook of our future research.

\section{Structure and Energetics of Adsorbates on Surfaces from First-Principles Calculations}

\subsection{Water adsorption on $\mathrm{Ru}(0001)$}

Aside from its relevance in heterogeneous catalysis, electrochemistry and corrosion, water adsorption on metal 
surfaces is a key topic to explore a number of fundamental issues in molecular and solid state physics, as for instance molecular cluster formation [27] and the ferroelectricity of water ice [28-30]. In addition, the great abundance of water, ice and water-covered solid surfaces in the biosphere explains the attention devoted to the study of water adsorption on single crystal surfaces by means of modern surface science techniques [31,32].

In particular, for $\mathrm{H}_{2} \mathrm{O} / \mathrm{Ru}(0001)$, the possible structures water can form, from adsorbed isolated molecules to small clusters, periodic bilayers or ice multilayers were extensively studied during 20 years since the late 1970s through a wide variety of experimental techniques [27, 33-39]. The basic findings were: (i) three distinct water peaks in the thermal desorption spectra (TDS), one at low temperature $(150 \sim 160 \mathrm{~K})$ attributed to ice multilayers, and two at higher temperatures $(170 \sim 180 \mathrm{~K}$, $210 \sim 220 \mathrm{~K}$ ) attributed to water aggregates (periodic bilayers, clusters) in more direct contact with the metal surface; (ii) an ordered $(\sqrt{3} \times \sqrt{3}) \mathrm{R} 30^{\circ}$ low energy electron diffraction (LEED) pattern at intermediate coverages; (iii) an isotope effect in $\mathrm{H}_{2} \mathrm{O} / \mathrm{D}_{2} \mathrm{O}$ desorption.

From this amount of experimental information at hand until 1982, the system appeared to be well understood, and a widely accepted model for water adsorption was proposed [34]. This model was based on the close match between the (0001) lattice of the Ru crystal and the hexagonal phase of ice (ice $I_{h}$, [40]), giving rise to the highest temperature peak (the "chemisorbed" state) in the thermal desorption spectra. The model further assumed a tetrahedral arrangement of water molecules as in ice $I_{\mathrm{h}}[40,41]$, and it involved, for the first bilayer, binding of the $\mathrm{H}_{2} \mathrm{O}$ molecules on top of the $\mathrm{Ru}$ atoms via one of the oxygen lone pairs and successive binding between these molecules and second layer molecules through at least two hydrogen bonds per molecule. This leads to the LEED $(\sqrt{3} \times \sqrt{3}) \mathrm{R} 30^{\circ}$ superstructure, with an ideal coverage $\theta=2 / 3$ for the first two layers, with the unit cell containing three $\mathrm{Ru}$ atoms and two $\mathrm{H}_{2} \mathrm{O}$ molecules, one in the first layer and one in the second [31].

In 1994, Held and Menzel presented the first complete LEED-IV analysis of the water bilayer at the $\mathrm{Ru}(0001)$ surface, after adsorption at $150 \mathrm{~K}[19,42,43]$. The $(\sqrt{3} \times$ $\sqrt{3}) \mathrm{R} 30^{\circ}$ structure was again observed, but the vertical arrangement was substantially different from what was formerly believed: the bilayer buckling was found to be only $0.10 \AA$ instead of $0.96 \AA$ in ice $I_{\mathrm{h}}$. These measurements ruled out the picture of an ice-like water bilayer at the $\mathrm{Ru}(0001)$ surface, proposing instead a "puckered monolayer", with a substantial distortion of the ideal tetrahedral angles in the hydrogen-bonded water molecular network.
The interest in the water bilayer on $\mathrm{Ru}(0001)$ was renewed by DFT-GGA calculations presented by Feibelman in 2002 [18]. Geometry optimization for the $\mathrm{H}_{2} \mathrm{O} / \mathrm{Ru}(0001)$ undissociated bilayer did not provide the LEED-IV measured buckling, and adsorption energetics was at variance with the thermodynamic criterion for wetting, i.e. adsorption energy greater than ice sublimation energy. Partially dissociative water adsorption at $\mathrm{Ru}(0001)$ was proposed as new model reconciling theory and experiments [18,44]: a partially dissociated bilayer showed geometry parameters in better agreement with the LEED-IV results, with the wetting condition fulfilled. While intrinsic discrepancies between this theoretical model and previous experimental evidences had been pointed out [44], a second theoretical work supported the half-dissociated bilayer picture [45], and vibrational features for this model were calculated [46]. The latter were subsequently investigated with sum frequency generation (SFG) vibrational spectroscopy [47], and a comparison with previous SFG studies of intact $\mathrm{D}_{2} \mathrm{O}$ interfaces $[48,49]$, strongly suggested that the water bilayer at $\mathrm{Ru}(0001)$ is not dissociated.

A number of important issues remained to be clarified, e.g. the assignment of the TDS peaks, the interpretation of the workfunction change upon adsorption, and the nature of the isotope effect [27,33-35,37,39,42], and we addressed these questions by means of firstprinciples calculations for the first bilayer of water at $\mathrm{Ru}(0001)$, considering several alternative structures. For these equilibrium geometries also details of the electronic structure were given, namely, the projected density of states (PDOS), the electronic charge density change, $\Delta \rho$, and the dipole moment change, $\Delta \mu$, upon adsorption. The aim was to provide a sound basis for this discussion, by treating all the structural alternatives with one and the same method, namely, gradient-corrected DFT, and to exclude possible artefacts of the method by pushing the calculations to an accuracy as high as possible. This was achieved by using $\mathrm{Ru}$ slabs thicker than was previously done, with adsorption at both slab sides and no atom constraints in the geometry optimizations. Electronic structure calculations and geometry optimizations were done within the DFT formalism with the help of the Cambridge Serial Total Energy Package (CASTEP), employing a supercell geometry [50,51] and the generalized-gradient approximation (GGA), in the form given by Perdew and Wang [52]. The outer 16 electrons for $\mathrm{Ru}$ (i.e. $4 s, 4 p, 5 s, 4 d$ ), and the valence electrons for $\mathrm{O}$ and the $\mathrm{H}$ electron were explicitly considered, using a plane-wave basis set, and the ionic cores of the $\mathrm{Ru}$ and $\mathrm{O}$ atoms were represented by scalar-relativistic [53,54], fully separable, ultrasoft [55] pseudopotentials. Despite the absence of core electrons for the hydrogen 

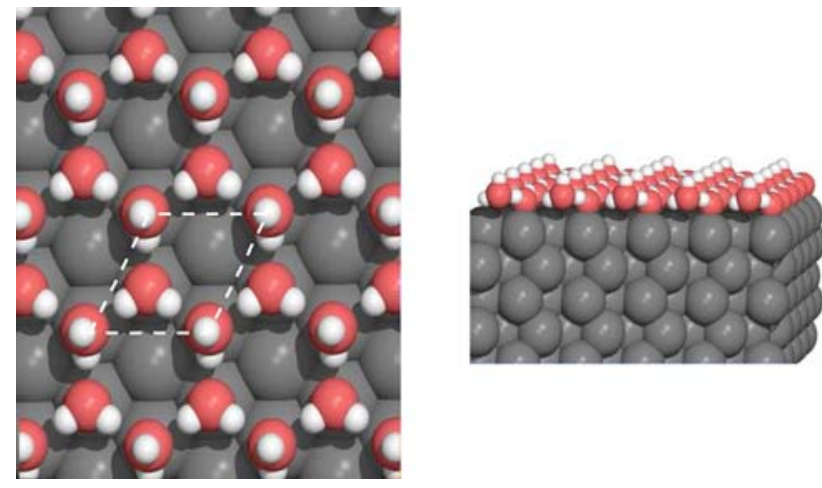

Fig. 1 Top (left) and side (right) view of the $\mathrm{H}$-up $\mathrm{H}_{2} \mathrm{O} / \mathrm{Ru}(0001)$ bilayer, with the $\mathrm{p}(\sqrt{3} \times \sqrt{3}) \mathrm{R} 30^{\circ}$ cell indicated

atom, its nucleus was also represented by the same kind of pseudopotential [55], which reduced the energy cutoff for the plane-wave basis set. Extensive test calculations on $\mathrm{Ru}$ bulk, $\mathrm{O} / \mathrm{Ru}$ system, various water structures and hexagonal ice were used to examine convergence with respect to system and integration parameters [56].

We performed electronic structure calculations and geometry optimizations for three alternative structures for the water bilayer on $\mathrm{Ru}(0001)[18,19,45,47]$, namely, the "H-up", "H-down", and half-dissociated bilayers, using a symmetric nine-layer slab. For the half-dissociated bilayer we also examined the structure with no hydrogens adsorbed on $\mathrm{Ru}$ at the center of the $\mathrm{O}$ hexagons $[18,45]$.

Here, we first consider the standard model for the water bilayer at $\mathrm{Ru}(0001)$, where all the second layer molecules have their dipole moment with the $z$ component oriented out of the metal surface ("H-up" bilayer [34]), Fig. 1. This model corresponds to a 2-layer stack of the ice $I_{\mathrm{h}}$ structure [40]. Test calculations with the (large) $(3 \times 3)$ revealed that $\mathrm{p}(\sqrt{3} \times \sqrt{3}) \mathrm{R} 30^{\circ}$ was sufficiently reliable, and therefore this cell was consistently adopted for the other structures. In line with former DFT results on this structure $[18,45]$, the bilayer buckling and first layer distance from the $\mathrm{Ru}(0001)$ surface exceeded the experimental values, and the calculated adsorption energy for "H-up" $\left(\mathrm{E}_{\mathrm{ads}}=0.56,0.58 \mathrm{eV}\right)$ turned out $\sim 0.15 \mathrm{eV}$ smaller than our calculated value of the sublimation energy for ice $I_{\mathrm{h}}\left(\mathrm{E}_{\mathrm{subl}}=0.73 \mathrm{eV}\right)$. In our opinion this does not necessarily imply that such a bilayer could not form at all, since the sublimation energy for ice multilayer could be lower than that of bulk ice.

The "H-down" model (Fig. 2) has become subject of investigation after Feibelman's work [18], and the theoretical $[57,58]$ and experimental (XPS, XAS, XES) [58] evidences on the $\mathrm{H}_{2} \mathrm{O} / \mathrm{Pt}(111)$ system. Adsorption energy and geometry of the "H-down" bilayer showed
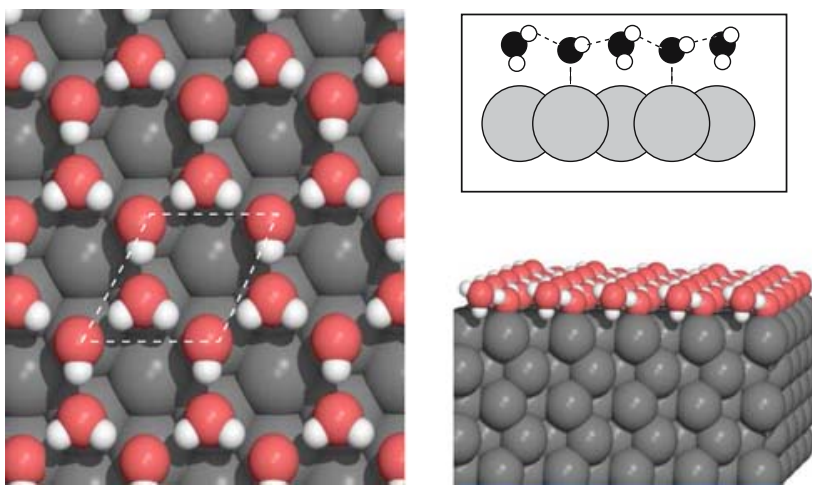

Fig. 2 Top (left) and side (right) views of the H-down $\mathrm{H}_{2} \mathrm{O} / \mathrm{Ru}(0001)$ bilayer, with the $\mathrm{p}(\sqrt{3} \times \sqrt{3}) \mathrm{R} 30^{\circ}$ cell indicated
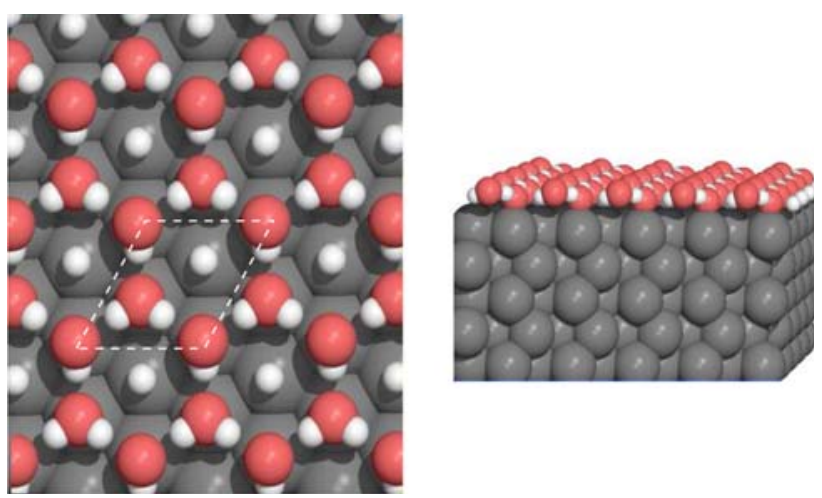

Fig. 3 Top (left) and side (right) views of the half-dissociated $\mathrm{H}_{2} \mathrm{O} / \mathrm{Ru}(0001)$ bilayer, with the $\mathrm{p}(\sqrt{3} \times \sqrt{3}) \mathrm{R} 30^{\circ}$ cell indicated

similarities to the "H-up" structure. In particular the adsorption geometry, the bilayer buckling and the $\mathrm{Ru}-\mathrm{O}$ distances turned out to be within few percent of the corresponding "H-up" parameters, i.e. again in partial disagreement with experiments. The "H-up" and "Hdown" structures differed significantly in the internal geometry parameters of the water molecules. Briefly, $\mathrm{H}$-down showed a particularly low value for the $\mathrm{HOH}$ angle in the second layer, a degeneracy in the three hydrogen bonds in the bilayer, and a longer "H-down" $\mathrm{OH}$ bond. We further considered a different "H-down" structure, what we called "H-down-rotated", which gave essentially the same results.

In the half-dissociated bilayer, as originally considered by Feibelman [18], (Fig. 3), every second layer water molecule has lost its non-hydrogen-bonding hydrogen, giving rise to a hydrogen-bonded hydroxyl, and the dissociated hydrogens are adsorbed in the same periodic structure on top of the naked $\mathrm{Ru}$ atoms in the center of the oxygen hexagons. Our results for this structure confirmed Feibelman's [18] and Michaelides et al.'s [45] findings. Specifically, we obtained (i) a value of bilayer buckling and $\mathrm{O}-\mathrm{Ru}$ distance considerably 
smaller than those of the intact bilayers, and in satisfactory agreement with the values obtained by the LEED-IV analysis [19,59], (ii) an adsorption energy $\sim 0.30 \mathrm{eV}$ higher than the one for the intact bilayers, and $\sim 0.15 \mathrm{eV}$ higher than the sublimation energy for ice $I_{\mathrm{h}}$. The lowering of the average bilayer distance from the surface with respect to the intact structures finds its justification in the substitution of a water molecule, which binds the surface via long-range non-bonding interactions, with an $\mathrm{OH}$ radical, binding the surface through a real chemical bond [45]. Note that now the first layer is made of $\mathrm{OH}$ radicals and the second of intact molecules and that both the $\mathrm{H}_{2} \mathrm{O}$ molecule and the $\mathrm{OH}$ radical lie almost flat, with their dipole moment directed out of the surface. Also, the buckling of the $\mathrm{Ru}$ atoms underlying the two oxygen atoms is significantly smaller than in the intact bilayers, and than its experimental value [59]. The change in adsorption energy from the intact to the half-dissociated bilayer structures is not only due to the dissociation of an adsorbed water molecule but also due to the considerable approaching of the remaining undissociated water molecule to the surface. In addition, the rather large $\mathrm{O}-\mathrm{H}$ bond length in the water molecule indicates a hydrogen bond strengthening with respect to the intact bilayers. We further note that our calculations predict an $\sim 0.15 \mathrm{eV}$ larger binding energy $E_{\text {ads }}$ than the one found in $[18,45]$, the latter being possibly due to the use of thicker slabs with respect to $[18,45]$.

We have also investigated an alternative halfdissociated structure $[18,45,59]$ where the dissociated hydrogens are removed from their on-top positions at the center of the hexagons and let adsorb at fcc sites [60] in a separate $\mathrm{p}(\sqrt{3} \times \sqrt{3}) \mathrm{R} 30^{\circ}$ cell. Although, as pointed out in Ref. [45], the existence of such system depends both on the presence of clean patches of $\mathrm{Ru}(0001)$ surface and on the ease of $\mathrm{H}$ diffusion throughout the halfdissociated bilayer, the recent LEED analysis of Puisto et al. [59] had given a considerable hint in favor of it. We did not find significant differences with the original Feibelman's model as what concerns the bilayer buckling and $\mathrm{O}-\mathrm{Ru}$ distance $[18,45,59]$, but we found some differences in the total $\mathrm{Ru}$ buckling, which turned out smaller than the one in the previously analyzed halfdissociated structure, and also than the value of the bestfit LEED geometry [59]. Another considerable difference was in the adsorption energy, that was $\sim 0.30 \mathrm{eV}$ greater than the one obtained by placing $\mathrm{H}$ at the center of the hexagons, probably due to the higher adsorption energy of $\mathrm{H}$ at the fcc sites with respect to on-top sites on $\mathrm{Ru}(0001)$ [60].

Based on this geometric-energetic results we confirmed that Feibelman's half-dissociated structure was the best candidate for explaining the experimental results. However, the detailed study of electronic structure, put some doubts on this interpretation. Analysis of the electronic charge density change upon adsorption $\Delta \rho_{\text {ads }}$ revealed that the way the electronic charge is redistributed upon adsorption is drastically different in the half-dissociated bilayer with respect to the intact ones. For the half-dissociated bilayer the bond of the second molecule $(\mathrm{OH})$ to the Ru surface is of the same kind (charge transfer from the surface to the adsorbate) as for the first molecule $\left(\mathrm{H}_{2} \mathrm{O}\right)$, whereas in the intact bilayers it is of opposite kind. These differences translate into marked differences also for the change of the surface dipole moment $\Delta \mu$ upon adsorption. When an adsorbate releases electron charge to the surface, $\Delta \bar{\rho}_{\text {ads }}(z)$ is $>0$ close to $z=0$ and $<0$ at high $z$ values, giving $\Delta \mu_{\mathrm{T}}<0$, whereas when an adsorbate subtracts electron charge from the surface the situation will be opposite, and $\Delta \mu_{\mathrm{T}}>0$. This is consistent with the fact that in the former case the adsorbate generates a surface dipole opposite to the one produced by the spill-out of the surface electrons, whereas in the latter case the adsorbate-induced dipole adds itself to the intrinsic dipole of the surface. We got $\Delta \mu_{\mathrm{T}}$ ("H-down") $=-0.35 \mathrm{D}<\Delta \mu_{\mathrm{T}}$ ("H-up") $=-0.16 \mathrm{D}<\Delta \mu_{\mathrm{T}}$ ( single layer) $=-0.05 \mathrm{D}$ $<\Delta \mu_{\mathrm{T}}$ (half-dissociated) $=+0.40 \mathrm{D}$. So the different charge redistribution upon adsorption in the half-dissociated bilayer with respect to the intact structures generates a $\Delta \mu_{\mathrm{T}}$ of opposite sign. Adding the adsorbate dipole contribution $\left(\Delta \mu_{\mathrm{I}}\right)$ to the surface dipole change, we concluded that the surface dipole change upon adsorption $\Delta \mu$ will be negative for the intact bilayers (larger for the "H-up", considerably smaller for the "H-down"), and weakly positive for the half-dissociated bilayer. This has implications also on the behavior of the workfunction change upon adsorption $\Delta \phi$, in the different structures, that experiments on the water bilayer on $\mathrm{Ru}(0001)$ predict to be significantly negative $(\sim-1.3$ to $-1.4 \mathrm{eV}$ $[39,42,61,62])$. A negative $\Delta \phi$ can sensibly be associated with a negative $\Delta \mu$, and vice versa: if the adsorbate decreases the surface dipole $(\Delta \mu<0)$, the valence electrons can be more easily extracted from the surface $(\Delta \phi<0)$. Thus the intact structures, showing a charge transfer from the adsorbate to the surface, are the best suited to explain the experimentally found $\Delta \phi \ll 0$ $[39,42,61,62])$, whereas the half-dissociated structures, showing a charge transfer from the surface to the adsorbate, are more compatible with $\Delta \phi>0$.

Hence, the geometric and energetics structure supported the half-dissociated models, whereas details of the electronic structure turned out in much better agreement with $\Delta \phi$ measurements for the intact models. In addition, independent calculations on the barrier height to dissociation [45] were in line with an intact adsorption 
model. This controversy could be due to either the inaccuracy of the DFT or electron-induced effects which, though ruled out in the LEED-IV works by Held and Menzel [19,42], were worth considering in light of recent experimental work [63] on X-ray damage in XPS measurements.

Soon after this work was completed, a number of insights came from additional theoretical and experimental works. Feibelman [64], using $\mathrm{Ar}$ as an $\mathrm{H}_{2} \mathrm{O}$ surrogate, quantified the error of DFT-GGA in describing the weak bonding of the second layer water molecule to the surface in the intact structures, and ruled out DFT inaccuracies in the relative stability of the above mentioned structures. Faradzhev et al. [65] reported very high cross sections and low threshold energies for electron impact induced dissociation of water molecules, which supports the beam damage hypothesis in previous experimental works $[19,42]$. Very recently, a combined experimental and theoretical effort [66] proved unambiguously that intact water may grow on $\mathrm{Ru}(0001)$ and explained a number of seemingly contradicting results with a new model for water adsorption. In this model, chains of flat lying (the preferential arrangement for single-molecule adsorption) and "H-down" water molecules are embedded in a honeycomb network of hydrogen bond water, retaining the hexagonal, honeycomb oxygen backbone consistent with the $(\sqrt{3} \times \sqrt{3}) \mathrm{R} 30^{\circ}$ LEED pattern, satisfying the thermodynamic wetting criterion, and giving rise to a work function consistent with the experimental value. Could it be the end of the story?

\subsection{Oxygen adsorption on $\mathrm{Al}$}

Oxidation of metal surfaces is a phenomenon of great importance in various fields from heterogeneous catalysis to corrosion [8]. The formation of oxides which lead to passivation and depassivation of surfaces [67] is a complex multistage reaction that is not completely understood at molecular level.

When exposed to oxygen aluminum forms a thin layer of $\mathrm{Al}_{2} \mathrm{O}_{3}$ that presents relatively good corrosion protection. Aluminum is very corrosion resistant in neutral or moderately acid environments ( $\mathrm{pH} 4-9)$ and can be used in many cases without any special protective coating or surface treatment [68]. In highly acid or basic environments, the rate of corrosion is high and the use of aluminum should be avoided. But with special anodizing processes modification of the surface is possible, like formation of colored, wear resistant and low friction surfaces [69]. For these motivations the study of the interactions of $\mathrm{O}_{2}$ with $\mathrm{Al}$ surfaces is of great interest to get a detailed understanding of the formation of a protective film of oxide, and this has been the subject of several theoretical and experimental studies. However, oxygen dissociation over a clean aluminum surface is still not well modeled.

In this context, the interaction of oxygen with the (110) surface of aluminium has been considered as a model case for metal oxidation. For this open surface, the available experimental data [70] suggest that formation of a disordered oxide structure occurs directly, without intermediate steps. No reliable experimental data are available for the binding energies of the oxygen atoms at the adsorption sites of $\mathrm{Al}$ surfaces, but they are estimated to be comparable to values observed for transition metals [71]. On this basis, one can explain the long relative distance reached by hot $\mathrm{O}$ adatoms before being adsorbed at the hollow site on the $\mathrm{Al}$ surface (see [72] and reference therein).

We studied the first step of oxygen dissociation on $\mathrm{Al}(110)$ (clean) surface [73], by means of plane-wave DFT methods in the generalized-gradient approximation $[74,75]$ and projected augmented wave (PAW) [7679], as coded in the Vienna ab-initio Simulation Package (VASP) [80]. A slab supercell approach was employed to model the $\mathrm{Al}(110)$ substrate, using a $p(2 \times 2)$ surface unit cell for all coverages, and a five layers slab, taking into account surface dipole correction and spin polarization effects.

We considered different approaches of the $\mathrm{O}_{2}$ molecule to the $\mathrm{Al}(110)$ surface along pathways perpendicular to the surface through high symmetry adsorption sites, that is, the top (T), bridge (B) and hollow (H) sites. We also investigated the energetics of oxygen atoms located above and below these sites, for different oxygen coverages, $\theta=1,0.5$ and 0.25 . Finally, we also performed several calculations for hybrid systems with above-surface as well as sub-surface oxygen atoms. Interaction energies and work function changes upon adsorption will be reported in a forthcoming publication [73].

For the molecule we considered 12 different adsorption channels that present the molecule on top, bridge and hollow sites, perpendicular and parallel to the surface. The calculations of these constrained channels were quite straightforward but location of the minima was tedious so that was evident that the molecular adsorption is unfavorable. When geometry optimizations are launched from the metastable point of the surface, a rich energy landscape emerges. Our results indicate that, when the $\mathrm{O}_{2}$ molecule reaches the surface, direct, barrierless dissociation is very likely to occur on almost all the considered pathways, in agreement with the experimental observation. Indeed, experimental evidence indicates that $\mathrm{Al}$ surfaces are so eager of oxygen that, under normal conditions, $\mathrm{O}_{2}$ molecules readily dissociate when exposed to this metal. With few exception, two types of 


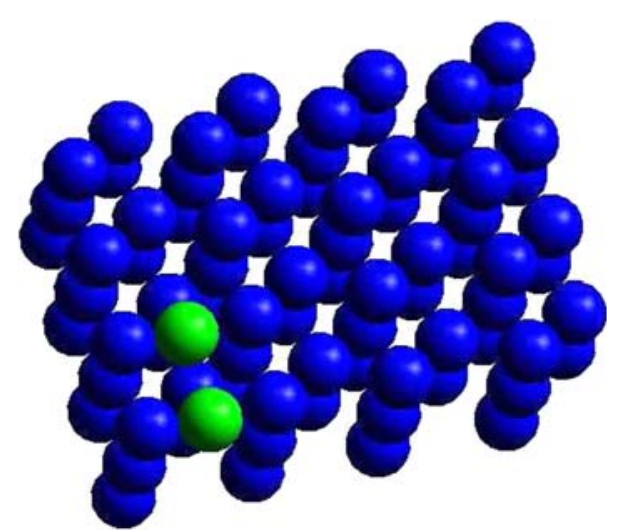

Fig. $4 \mathrm{O}-\mathrm{Al}$ network, with the oxygen atoms linked to three atoms of the metal. Oxygen atoms are represented by green spheres

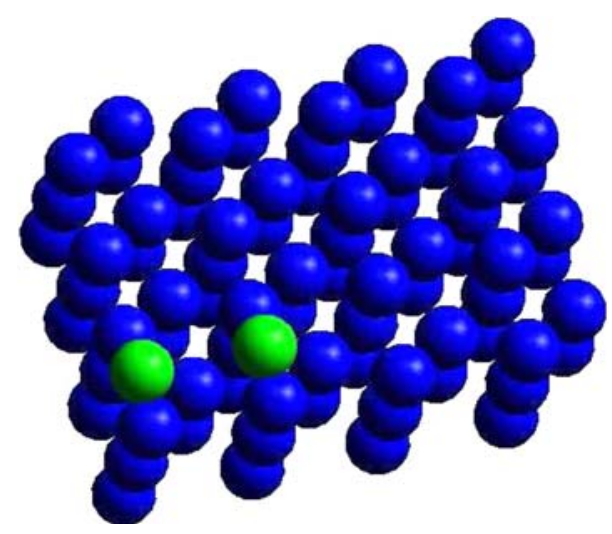

Fig. 5 O-Al-O chains along the [001] directions on the surface. Oxygen atoms are represented by green spheres

arrangements are observed, that is (i) an $\mathrm{O}-\mathrm{Al}$ network, with the oxygen atoms linked to three atoms of the metal (Fig. 4), and (ii) O-Al-O chains along the directions of the surface (Fig. 5).

For what concerns atomic oxygen, we found that for all coverages octahedral hollow sites on the surface are the most stable adsorption sites $(4.1-4.3 \mathrm{eV})$ whereas top sites are the less stable ones $(\lesssim 1.4 \mathrm{eV})$. The high adsorption energy values found for hollow and bridge sites are comparable to those of $4 \mathrm{~d}$ transition metals [81]. At low coverage, we observed that oxygen atoms tend to move laterally from the center of the adsorption cavity to its border, in order to optimize the interaction with two metal atoms. When coverage is 0.5 the most stable geometry presents the same arrangements observed for the dissociative chemisorption of the molecule (Fig. 4,5). At high coverage, we found considerable reconstruction of the surface, with formation of $\mathrm{O}-\mathrm{Al}$ domains. $\mathrm{O}-\mathrm{Al}$ equilibrium geometry and adsorption values compare favorably with experiments [70].

We found stable absorption sites between the first and second layer below all surface sites but, with the excep-

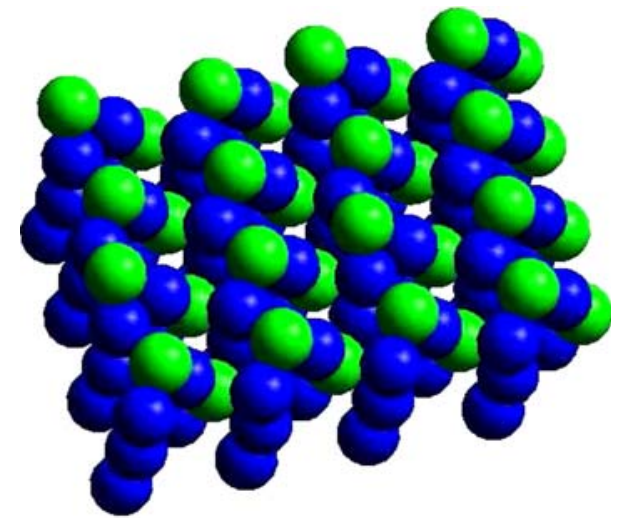

Fig. 6 Formation of an $\mathrm{Al}_{2} \mathrm{O}_{3}$ phase when $\Theta=2$. Oxygen atoms are represented by green spheres

tion of the top site, they are less stable than the abovesurface ones. When the $\mathrm{O}$ atom is absorbed in a subsurface top site, it is located at the center of an octahedral cavity, about $2 \AA$ below the surface, and with adsorption energies ranging from 3.6 to $4.1 \mathrm{eV}$, depending on coverage. We further considered hybrid systems with subsurface monolayer as well as on-surface oxygen atoms: when the full bilayer coverage is reached a convergence of the adsorption energies, for all sites, around $4 \mathrm{eV}$ is found.

From the analysis of the stable geometries we notice that increasing of coverage results in thickening of $\mathrm{AlO}$ chains, with formation of an $\mathrm{Al}_{2} \mathrm{O}_{3}$ (see Fig. 6) phase when $\theta$ reaches the bilayer value. This suggests that oxygen adsorption results in formation of localized $\mathrm{O}-\mathrm{Al}$ bonds at low coverage, followed by considerable surface reconstruction and formation of $\mathrm{Al}_{2} \mathrm{O}_{3}$ phase. This is in agreement with the observation of Mitchell et al. [82] who noticed two distinct steps in aluminum oxidation mechanism, for all three low-index surfaces. Geometrical parameters are in agreement with this mechanism: the two shortest $\mathrm{Al}-\mathrm{O}$ bond lengths [83] in $\alpha-\mathrm{Al}_{2} \mathrm{O}_{3}$ are $1.86 \AA$ and $1.97 \AA$, and the unrelaxed octahedral-site bond length is $2.025 \AA$.

We may therefore conclude that oxygen chemisorption on aluminum is much more complicated than it might appear at first glance, and dynamical studies are needed for a correct understanding of the passivation phenomenon.

\section{Classical Studies of Dynamical Processes at Surfaces}

3.1 Eley-Rideal vs. hot atom dynamics in hydrogen recombination on $\mathrm{Ni}(100)$

Recombination reactions on surfaces may occur through a number of distinct mechanisms. Traditionally [8], one 
distinguishes between Eley-Rideal (ER) and LangmuirHinshelwood (LH) reactions. In the ER mechanism an atom from the gas-phase directly scatters off a previously adsorbed atom and forms the reaction product. In the LH mechanism both reactants adsorb on the surface, thermally diffuse on it and encounter each other to form the reaction product. The latter mechanism dominates at medium to high temperatures [84], but it is limited at low temperature by the thermal diffusion of the reagents, which behaves like a typical activated process [72]. In addition, for some systems LH reactions are endothermic and therefore energetically forbidden at low temperatures, whereas ER processes are typically exothermic. Thus, for example, since hydrogen adsorption energies on transition metal surfaces are in the range $2.5-2.8 \mathrm{eV}$, formation of $\mathrm{H}_{2}$ molecules $\left(D_{0}=4.5 \mathrm{eV}\right)$ on such surfaces is endothermic by $0.5-1.1 \mathrm{eV}$ within the LH scheme, and exothermic by $1.7-2.0 \mathrm{eV}$ within the ER scheme. Signatures of the LH mechanism had been readily available since its introduction in the 1920s. On the other hand, despite its remarkable fingerprints, experimental observation of the direct, non-thermal, Eley-Rideal reaction had to wait the detailed measurements of Rettner et al. on the $\mathrm{H}+\mathrm{H} / \mathrm{Cu}(111)[85,86]$ and on the $\mathrm{H}+\mathrm{Cl} / \mathrm{Au}(111)$ systems [87], where a direct component was clearly resolved in the time-of-flight spectra of the product molecules. What these measurements could not resolve was the occurrence of a third, Hot-Atom (HA) mechanism. This mechanism had been previously suggested by Harris and Kasemo [88] and it is intermediate between the above two: one of the two reagents from the gas-phase is not fully accommodated on the surface, rather diffuses on it at hyperthermal energy until it encounters its reaction partner. Evidences for an HA mechanism came from observation that measured "ER" cross sections $[85,86]$ were an order of magnitude greater than those theoretically predicted [89-91]. Hot secondary precursors are needed to explains $\mathrm{D}_{2}\left(\mathrm{H}_{2}\right)$ formation in kinetic $\mathrm{H}(\mathrm{D})$ abstraction experiments on a variety of $\mathrm{D}(\mathrm{H})$-covered metal and non-metal surfaces [92-102] at low temperature. They could even be unambiguously distinguished in angularly resolved kinetic experiments in $\mathrm{H}$ on $\mathrm{D}$ abstraction on $\operatorname{Pt}(111)$ [101,102], though the contribution of ER mechanism remained elusive to quantify. Hot atoms may also form upon strongly exothermic dissociative chemisorption, and indeed the earliest indication of HA processes came from dissociative chemisorption of $\mathrm{O}_{2}$ on $\mathrm{Al}(111)$ [72].

There is clearly much interest in the HA route, since it is limited neither by the smallness of the ER cross section nor by the (thermal) diffusion of the reagents. It is now generally accepted that hot atoms may form even

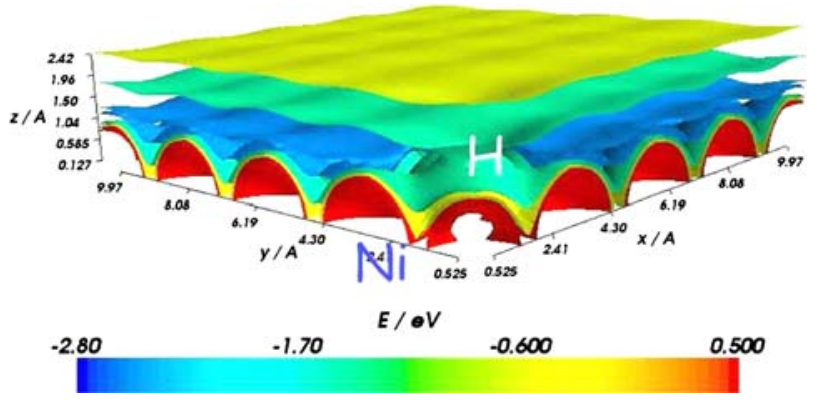

Fig. 7 Isosurfaces representing the interaction potential of an hydrogen atom on an $\mathrm{Ni}(100)$ surface. $\mathrm{Ni}$ atom positions can be deduced by the form of the high energy isosurfaces ( red). A hydrogen atom (at rest) has been put in the front corner in order to show the corrugation induced by the presence of an adsorbate

with heavy species [72], and with hydrogen atoms they are common on metals where they experience strong, non-directional attraction. This is illustrated in Fig. 7 where we represent an isosurface map of the interaction potential between a hydrogen atom and an $\mathrm{Ni}(100)$ surface, as derived by the EDIM potential developed by Truhlar et al. [20,21].

Much theoretical work had been done in studying HA dynamics, particularly in the context of hydrogen recombination on metal surfaces. We performed classical trajectory calculations on $\mathrm{Ni}(100)$ in the single adsorbate case at normal incidence, in order to quantitatively compare the efficiency of HA formation with that of the ER reaction [22]. We used our own gas-surface code TRAJ (see [103,104]), which was properly modified [22] to include the EDIM potential model $[20,21]$ and the treatment of ER/HA dynamics to be discussed in the following. In particular, we compared ER reaction cross sections and HA formation cross sections both with a rigid and a non rigid Ni substrate over a wide range of collision energies, with some emphasis on the energy transfer issue. Subsequent studies will address the question of how this efficiency translates into the overall formation of the diatomic molecule.

The rigid surface model was a benchmark study in which truly bound hot atoms formed with a finite cross section as a consequence of the energy exchange process which occurs between the projectile and the target atom. When the energy transfer to the adsorbate atoms is negligible (e.g. for scattering at large impact parameter) projectile atoms can at most be in an unstable state in which they are only temporarily trapped to the surface, i.e. they bind to the surface until a corrugation induced energy transfer process (a collision with a lattice site) brings them back to the gas phase. These "metastable" HAs were characterized in terms of initial formation cross sections and residence times, although the first 

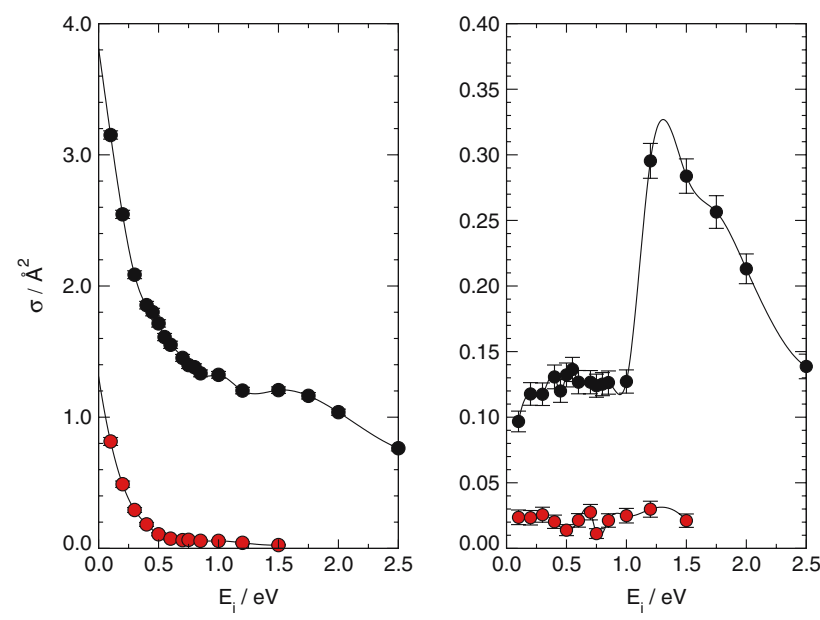

Fig. 8 Cross-sections results for the rigid-surface model. Left (projectile) hot atom formation cross section from occupied and nearest neighbor (nn) cell (black and red symbols, respectively). Right ER cross section (black circles) and contribution to the reaction cross section from the n.n. cell (red)
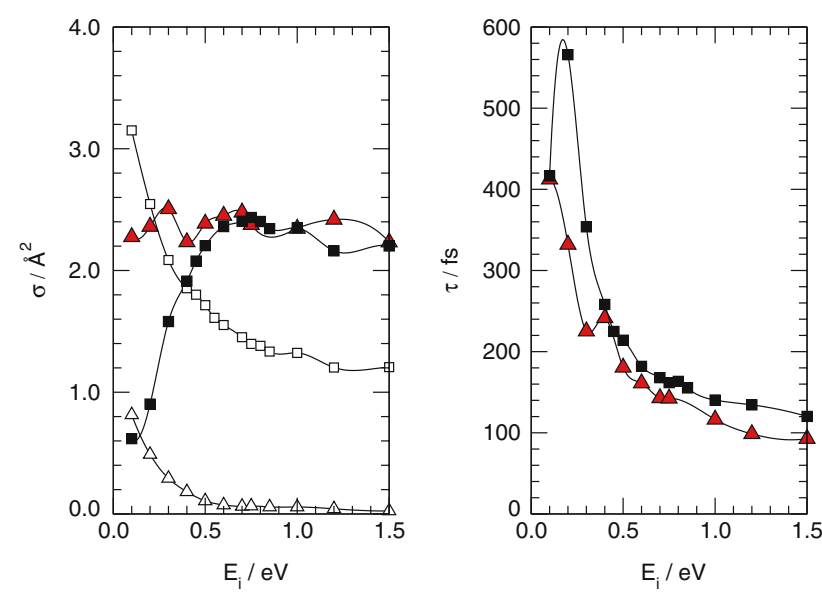

Fig. 9 Left panel metastable HA formation cross sections (per cell) for impact on the occupied and nn cell (black and red symbols, respectively), along with the results of Fig. 8 for comparison (open symbols). Right lifetime for metastable hot atoms (colours as in left panel)

cannot be a finite quantity in this case because empty sites contribute to the formation process. Figure 8 (right panel) clearly shows that ER cross section is quite small, and essentially constant $\left(0.11-0.12 \AA^{2}\right)$ for energies in the range $0.10-1.0 \mathrm{eV}$, because the strong accelerating potential reduces the role of the asymptotic kinetic energy of the projectile. It is much smaller than that corresponding to the formation of hot-atoms (left panel): projectile HA cross section can be $>30$ times greater than ER cross section, especially at low collision energy, where trapping of the incident atom reaches its maximum value. At higher collision energy hot atoms still form, but this time in a metastable state, Fig. 9. When the projectile impinges on the occupied cell the formation process depends on the collision energy because energy transfer to the adsorbed atom causes competition with the formation of bound species; on an empty cell, cross section for formation does not depend on the collision energy. Lifetimes do not depend on where hot atoms form, except in the low collision energy regime. Their behavior as a function of the incident energy is consistent with a corrugation induced desorption of the atoms: the higher the collision energy the higher is the kinetic energy of the traveling atoms, i.e. the higher is the collision frequency with lattice sites.

Before considering the more realistic non-rigid surface model, a few comments about the energy transfer issue are worth doing. Indeed, despite the unfavorable mass ratio between hydrogen and substrate atoms, projectiles are subjected to strong acceleration $(\gtrsim 2.5 \mathrm{eV})$ before impinging on the surface. This causes a substantial local heating of the substrate (Fig. 10), which causes some problems with the application of the usual periodic boundary conditions (PBCs). Indeed, while energy transfer to the bulk along the surface normal is taken into account with the help of Langevin atoms at the bottom of the slab, energy relaxation parallel to the surface is completely neglected and an artificial rise of the surface temperature is observed, since each periodic image of the slab is subjected to the same "collisional dynamics". This is clearly a problem if one is interested in the dynamical behavior of atoms traveling on the surface. Luckily, hot atoms travel across the surface at a much higher speed than that of the shock wave produced by projectile impact, i.e. they reach the slab edge before the arrival of that wave. We therefore developed a two-step algorithm to get rid of the problem due to the use of the PBCs: one follows HA dynamics up to the edge zone, stores coordinates and momenta of the atoms (and possibly those of the nearest neighboring substrate atoms) and starts a new dynamical simulation "seeding" the subsystem (hot atom + surrounding substrate atoms) into a slab at the initial temperature. This may be regarded as a "kinetic" approximation to the hot atom dynamics [22].

We used this two-step dynamical model in order to compute HA formation cross sections at a surface temperature of $120 \mathrm{~K}$, (Fig. 11). It is clear from the figure that: (i) HA formation cross section for impact on the occupied cell closely parallels the rigid-surface result, i.e. it mainly depends on the energy transferred to the target atom; (ii) energy relaxation is very slow, much slower than corrugation induced desorption; otherwise, some of the metastable HAs which occurred in the rigid surface model (Fig. 9) would be trapped onto a longlived state and increase the cross section values; and 
Fig. 10 Energy transfer to the substrate. Average kinetic temperature of some "shells" of first layer atoms as defined in the left inset for collisions at $0.10 \mathrm{eV}$ (left panel) and $1.0 \mathrm{eV}$ (right panel) (see text for details)
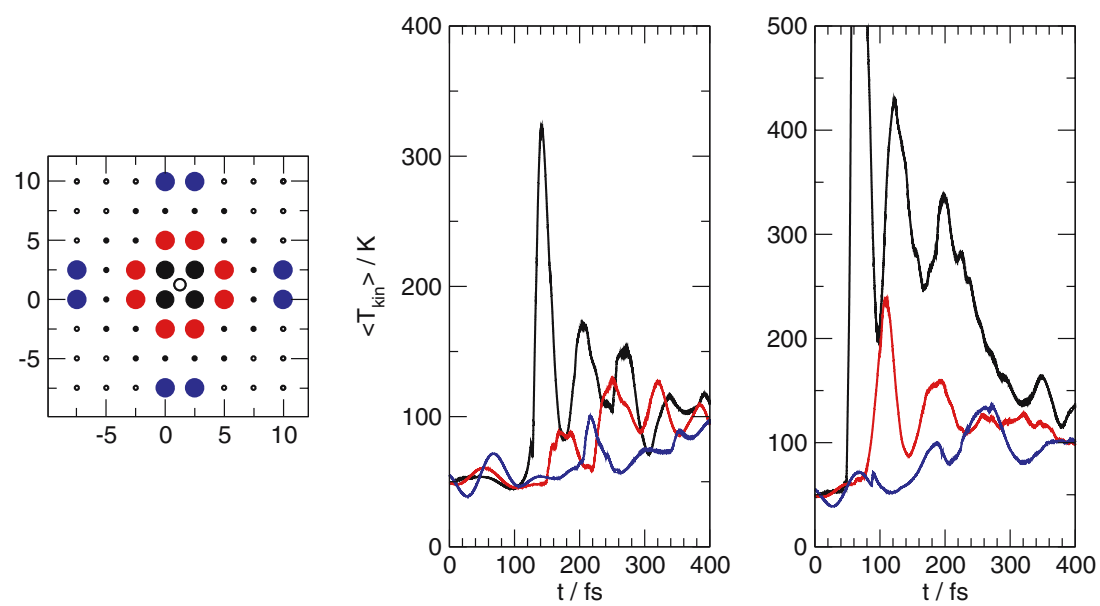

Fig. 11 Left formation cross sections for long-lived hot atom species. Center formation cross sections for metastable species. Right lifetime. Filled symbols for scattering at $T=120 \mathrm{~K}$ on the occupied (black) and empty (red) neighbor cells. Empty symbols are rigid-surface results for comparison (squares and triangles as before)
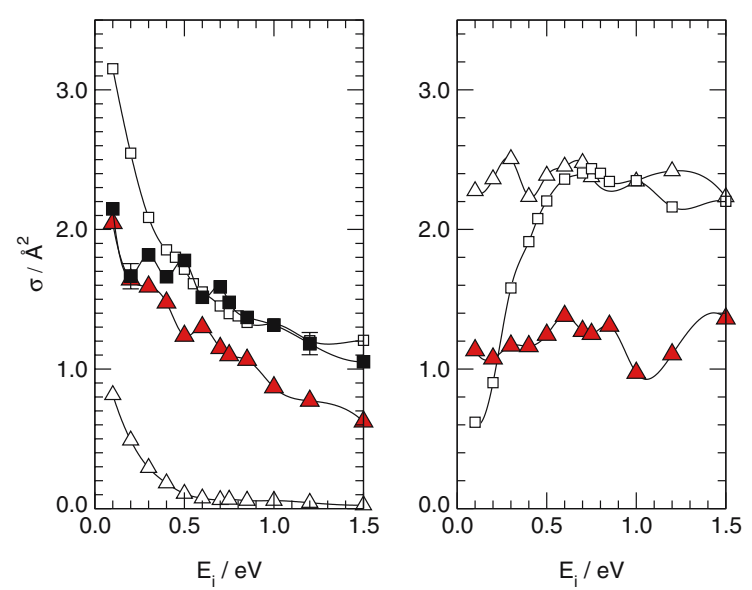

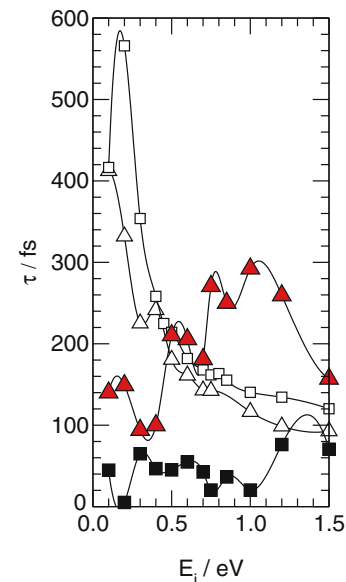

(iii) hot atoms form efficiently also when the projectiles imping on empty cells, thanks to energy transfer to the substrate atoms; note that when moving from the rigid to the non-rigid surface model the $\sim 1 \AA^{2}$ decrease $(\sim 50 \%)$ of the metastable formation cross section for scattering in an empty cell (center panel) is nicely accounted for by the increase of long-lived cross section (left panel). A more detailed account of hot atom properties and surface temperature effects can be found in the cited paper. Eley-Rideal results at $T=120 \mathrm{~K}$ show no appreciable change with respect to the rigid-surface results of Fig. 8, and a more detailed analysis suggests that target vibrations play a minor role in the recombination process, as long as energy dissipation to the lattice is included. ${ }^{1}$

\footnotetext{
1 Subsequent quantum mechanical calculations within the rigid, flat surface approximation revealed consistent vibrational enhancement of the ER cross sections, in agreement with quasiclassical trajectory studies including the zero-point-energy (ZPE). With a mobile substrate, addition of the ZPE is, in principle, not possible since relaxation may occur before the projectile impinges on the surface, when it starts from an arbitrary height above it.
}

3.2 Sticking and hot atom relaxation in dissociative chemisorption of $\mathrm{H}_{2}$ on $\mathrm{Ni}(100)$

Differently on what done in the previous subsection, here we consider dissociative chemisorption of hydrogen on a metal surface, the same $\mathrm{Ni}(100)$ face considered before, and HA formation in this process. Though less energetic than before, hot atoms may still form in such a process since the reaction exothermicity leaves atoms with $\gtrsim 0.25 \mathrm{eV}$ energy. Recently, these hot atoms have been theoretically investigated on a different metal surface, and their relaxation process was found very fast, much faster than simple impulsive arguments suggest [105]. Purpose of this (ongoing) research work is to characterize in details molecule dissociation and hot atom relaxation, using the above-mentioned EDIM potential model for the title system [20,21], which realistically allows to take into account surface atom motion.

As already mentioned above, hydrogen dissociative chemisorption on $\mathrm{Ni}(100)$ is exothermic, and is characterized by different activation barriers depending on the adsorbing sites and the orientation of the molecule 


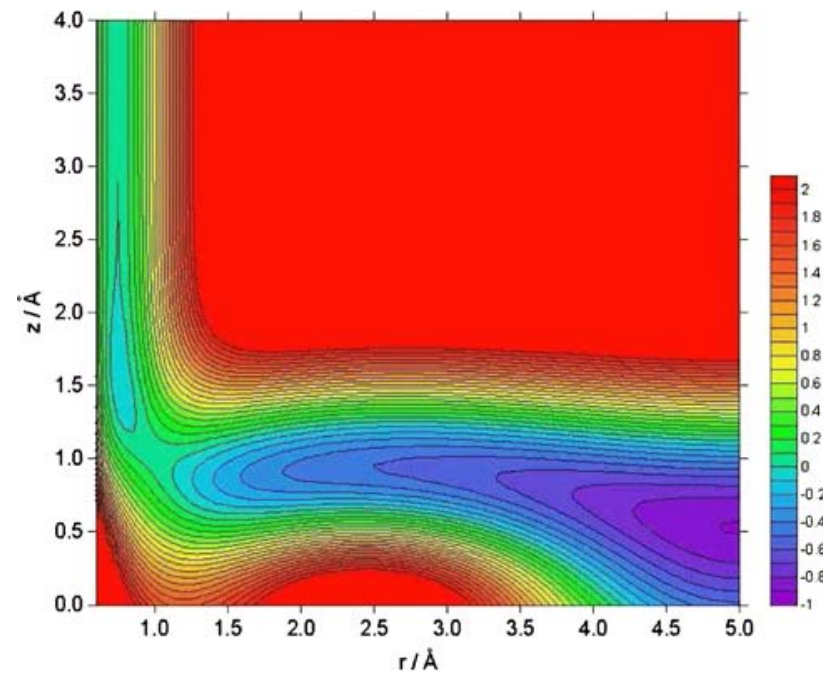

Fig. 12 Contour map of the potential energy surface for dissociative chemisorption of a hydrogen molecule on the hollow site of the Ni(100) surface. The molecular axis is kept parallel to the surface and directed toward the bridge sites. $r$ is the diatomic internuclear distance and $z$ is the center-of-mass height of the molecule. Energies in $\mathrm{eV}$, with respect to the equilibrium energy of isolated $\mathrm{H}_{2}$ molecule

with respect to the surface. With the potential model at hand, the energetically most favorable microscopic event occurs when a parallel oriented hydrogen molecule approaches a hollow site and, overcoming a small barrier, dissociates toward the bridge sites accommodating the two hydrogen atoms on the neighbor hollow sites, Fig. 12.

The dissociative probabilities of a hydrogen beam perpendicular to the surface have been calculated at several collision energies in the range $0.01-0.5 \mathrm{eV}$, (Fig. 13), using classical and quasi-classical trajectories and, as before, both a rigid and a non-rigid substrate. The pure classical trajectory results on the rigid surface (solid line) show an activated behavior with a dynamical threshold of $0.06 \mathrm{eV}$ which agrees with the aforementioned minimum energy path value, and the probability monotonically increases with the collision energy, as typically found for this process. It is worth noting that when the zero point energy of the hydrogen molecule is taken into account $(0.27 \mathrm{eV})$, dissociation is no longer activated-like, showing a decreasing behavior at low energies usually assigned to precursor states or steering of the incident molecules (circles). Hence, the vibrational energy is performingly channeled to the dissociation coordinate, as it is confirmed by the results for vibrationally excited molecules $[(v, j)=(1,0)]$ (squares). On the other hand, results show that thermal surface motion at a temperature of $50 \mathrm{~K}$ (dotted line) does not affect the dissociative sticking probability. This is due to the fact

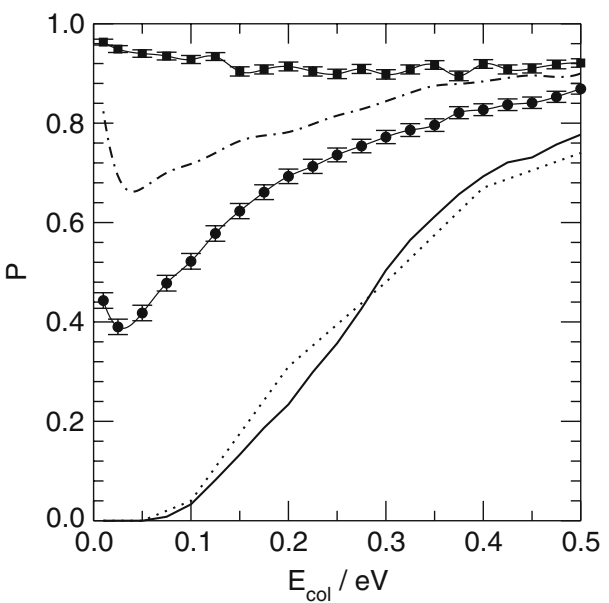

Fig. 13 Dissociative chemisorption probabilities of $\mathrm{H}_{2} / \mathrm{Ni}(100)$. Solid and dotted lines for classical trajectory calculations with a rigid and a non-rigid, $T=50 \mathrm{~K}$, surface. Filled symbols for quasiclassical results, for hydrogen molecules initially in the $(v, j)=$ $(0,0)$ (circles) and $(v, j)=(1,0)$ (squares) states. Dashed-dotted lines are rigid surface, classical-trajectory results with an EDIM potential model using $D_{\mathrm{e}}\left(H_{2}\right)=4.40 \mathrm{eV}$

that dynamics is direct, not involving intermediate precursor states, and dissociation occurs on a much shorter time scale than substrate vibrations; indeed, thermal lattice distortions play only a minor role in this essentially non activated process.

Because of its empirical origin, the available potential model is surely not accurate enough for reproducing experimentally available data on this system [106]. Even trajectory results employing first-princinples calculations of the interaction potential $[107,108]$ are at variance with experimentally determined sticking coefficients. We only note here the importance of a correct representation of the entrance channel of the reaction. Using the same EDIM potential as before, we can slightly modify it by changing the $\mathrm{H}_{2}$ binding energy, $D_{\mathrm{e}}$. Results obtained when decreasing $D_{\mathrm{e}}$ by solely $0.3 \mathrm{eV}$ (dashed-dotted line in Fig. 13) show how the shape of the sticking coefficient changes, from activated to unactivated sticking.

In order to quantitatively characterize hot atoms, we followed the time evolution of the $\mathrm{H}$ adatoms kinetic energies. On the rigid surface, whatever the incidence angle of the hydrogen beam, the trajectory-averaged kinetic energy of each adatom was found to be equidistributed in its spatial components (parallel and perpendicular to the surface) and constant as long as the origin of time is fixed by the condition that the interatomic distance is greater than $5 \AA$. These kinetic energy components show, of course, a linear dependence on the collision energy. 


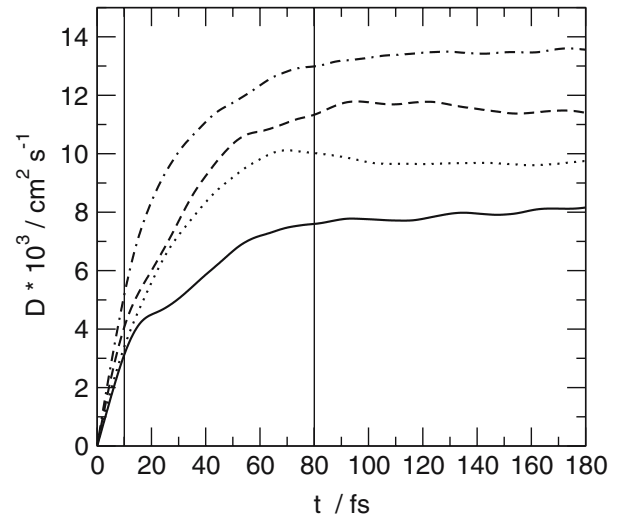

Fig. $14 D=\left\langle d^{2}\right\rangle / 4 t$ as a function of time for different initial collision energies of the impinging molecule normal to the surface. Solid, dotted, dashed and dashed-dotted lines for 0.1, 0.2, 0.3 and $0.5 \mathrm{eV}$, respectively. Vertical lines approximately mark the different regimes of hot atom motion: leftmost region is for ballistic motion and rightmost region for diffusive motion

After dissociation, the two $\mathrm{H}$ adatoms initially go through a ballistic motion along the surface and then, passing through a transition regime, they reach a hyperthermal diffusive regime. Note that this diffusive regime, due to surface corrugation, has to be distinguished with the usual thermal diffusion: random collisions with lattice sites cause reorientation of the momentum along the surface in an otherwise free-particle motion on the surface plane. This can be observed from the mean square displacement $\left\langle d^{2}\right\rangle$ of HAs from their "initial" position or, more clearly, from the value of the "instantaneous" diffusion coefficient, $D(t)=\left\langle d^{2}\right\rangle / 4 t$. [The true diffusion coefficient is given by $\left.\lim _{t \rightarrow \infty} D(t)\right]$. Here, as before, for each trajectory the hot atom is considered to be formed when the condition $r_{\mathrm{HH}}>5 \AA$ is fulfilled. We found that, whatever the collision energy, the time required for the ballistic-to-diffusive transition is found to be roughly constant ( $\simeq 70 \mathrm{fs}$ ), (Fig. 14). Results with a thermalized surface, as long as a detailed analysis of the influence of surface temperature, will be given in a forthcoming publication [109].

\section{Quantum Studies of Dynamical Processes at Surfaces}

As already said in the introduction, in recent years there has been an ever growing interest in the dynamics of hydrogen atoms on graphite surfaces. Apart from its importance in fusion reactors [110,111], and for the study of hydrogen storage in graphite nanostructures $[110,112,113]$, much of the current research has been motivated by the formation of hydrogen molecules in the ISM. It was hypothesized long ago that the molecular hydrogen abundance even in the most diffuse clouds
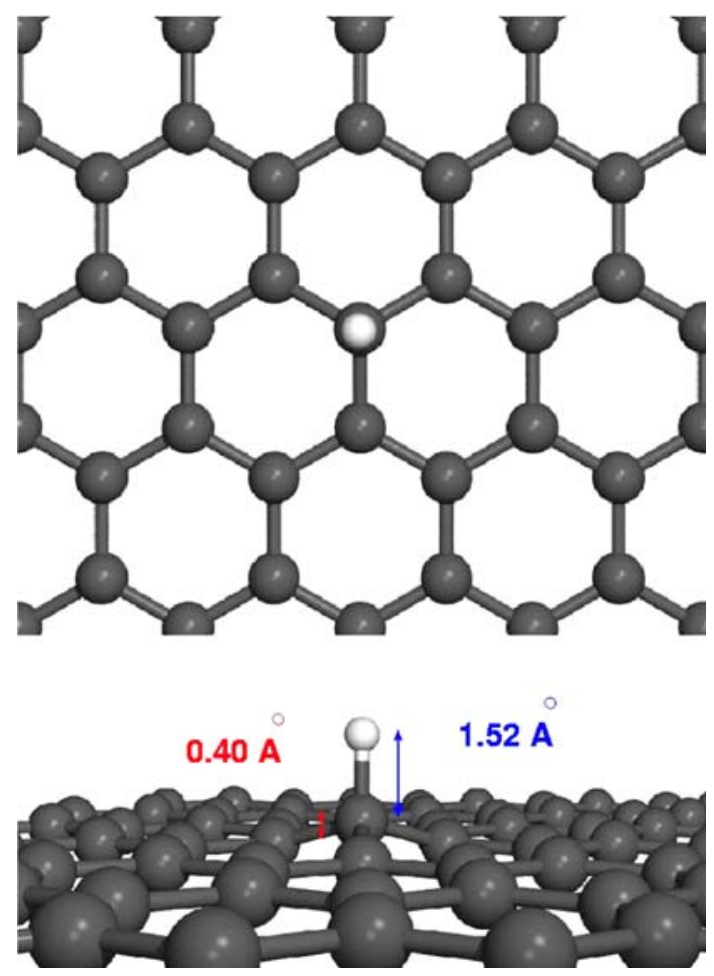

Fig. 15 Hydrogen chemisorption geometry on the C(0001) surface

(with $10^{2}$ atom per $\mathrm{cm}^{3}$ ) may only be explained by recombination on the surface of interstellar dust particles [114-116]. It is now generally accepted that cosmic dust is composed of very small particles of different sizes and nature [117-119]: in diffuse clouds, the largest particles (100 nm in size) are composed of a silicate core covered by an "organic refractory" mantle, whereas smaller particles are carbonaceous $(30 \mathrm{~nm})$ and even smaller particles are simply large molecules (polycyclic aromatic hydrocarbons, PAHs); in cold molecular clouds $\mathrm{H}_{2} \mathrm{O}$ and $\mathrm{CO}_{2}$ ice mantles are formed on the silicate core-organic refractory particles, and incorporate also the previously mentioned carbonaceous particles. Thus, at least in diffuse clouds, molecular hydrogen may be formed on carbon surfaces of dust particles, being either the refractory mantle or the smaller carbonaceous particles or PAH molecules. This has stimulated much of recent experimental and theoretical investigations on hydrogen interaction with graphite (0001).

In the ideal, defect-free graphite surface, hydrogen atoms may adsorb on the surface either chemically or physically. Electronic structure calculations [120-122] agree on the fact that hydrogen chemisorption occurs on top of a carbon atom and requires substantial lattice reconstruction, with the carbon beneath the $\mathrm{H}$ atom puckering out of the graphite plane by about $0.4 \AA$, Fig. 15. The carbon atom needs to rehybridize its valence 
orbitals, causing partial loss of the aromatic character of the graphite plane, and a barrier to chemisorption of about $0.2 \mathrm{eV}$ appears. It follows that subsequent adsorption on neighboring sites requires less energy, and formation of adsorbate pairs and quadruples occurs [123, 124]. Very recently, reaction of these pairs has been investigated with STM techniques [125]. The barrier indicates that sticking of $\mathrm{H}$ atoms in the chemisorption well hardly occurs - via a direct dynamics - at the conditions typical of diffuse clouds $(T \leq 100 \mathrm{~K})$, although it may occur for example in the so-called photo-dissociation regions [126]. On the other hand, physisorption of hydrogen atoms may occur at very low energy and leads to formation of weakly bound species far apart from the surface (at about $4 \AA$ ). In this case, DFT calculations are not accurate enough to describe the interaction and the physisorption binding energy has been estimated to be $\sim 40 \mathrm{meV}$ by selective adsorption experiments [127].

Several theoretical studies have been performed in the past on the Eley-Rideal [128-134] and LangmuirHinshelwood $[135,136]$ reactions. On the experimental side, chemisorption of hydrogen atoms has been studied [137] in conditions of relevance for photo-dissociation regions and good agreement was found with theoretical predictions [138]. Eley-Rideal reaction has been studied in laboratory $[139,140]$ and product rovibrational excitation has been found, although somewhat more limited than theoretically predicted. Some laboratory work has also been done on realistically analogues of interstellar granular surfaces like olivine (a mixed $\mathrm{Fe}, \mathrm{Mg}$ silicate) [141-143], amorphous carbon [143] and amorphous solid water films [144].

We used the potential model developed by Sha et al. [23], based on periodic DFT calculations [24], and a quantum scattering method in the rigid, flat surface approximation (see [26] and reference therein). We adopted different sets of coordinates in order to complement previous theoretical investigations on the Eley-Rideal reaction, considering the competitive CID process. The latter process had received some attention in the literature, both experimentally and theoretically $[145,146]$, but very little if any was known about the role of quantum dynamics (e.g. the quantization of the target-surface initial state) even when light atoms were involved. Thus our aim was twofold: (i) to study the CID of weakly physisorbed species, and (ii) to address the importance of quantum effects in such kind of process. It turned out the quantum effects were indeed found, mainly in the Eley-Rideal reaction involving chemisorbed hydrogen atoms $[25,26]$, and surprisingly that trapping of incident atom was efficient with physisorbed target atoms. In the following, we describe separately these results.
4.1 Quantum effects in Eley-Rideal hydrogen formation from chemisorbed species

Quantum effects in this hydrogen formation came out as a surprise since (i) they were found at relatively high collision energy, and (ii) the reaction is a simple, exoergic, barrierless reaction for which classical mechanics is expected to work well. Note that, though quantum effects undoubtedly exist for several collision systems, especially involving light atoms, their observation in collision experiments is quite difficult. For gas-phase systems, for example, quantization of the internal modes at the transition state (quantized transition states or quantized dynamical bottlenecks) or Feshbach resonances on purely repulsive potential energy surfaces are typical quantum effects that have been predicted for several collision systems, but that have been observed only for a few of them. The $\mathrm{H}+\mathrm{H}_{2}$ isotopic family of reactions is the best known example for quantized dynamical bottlenecks which lead to an observable time-delayed mechanism [147,148], and interference features [149,150]. The $\mathrm{F}+\mathrm{HD} \rightarrow \mathrm{HF}+\mathrm{D}$ reaction is the first reaction for which conclusive evidence has been found for a Feshbach resonance in a full collisional dynamics $[151,152]$ and only recently experimental evidence has been found for some polyatomic reactions [153-155]. We found clear quantum signatures in global observables like total EleyRideal cross sections, which indicate that some cautions are needed when applying classical methods to light atom dynamics, even when the reaction system, the reaction conditions or the observable to be computed suggest them to be adequate.

The computed quantum ER and CID cross-sections are shown in Fig. 16. In the left panel, the ER cross sections are reported in the energy range $0-2.5 \mathrm{eV}$ and in the right panel they are shown in a larger energy range along with the CID cross-sections. The results for the different values of $v$, the initial vibrational quantum number of the $\mathrm{H}$ atom adsorbed on graphite, are reported. Overall the ER cross sections are quite large, much larger than for hydrogen recombination on metals, e.g. see Fig. 8. This behavior is due to the much weaker substrate attraction which allows for steering of the incoming $\mathrm{H}$ atom, to be compared to the strong projectiles attraction on metal surfaces. In addition, the weaker chemisorption potential puts the target atom farer away from the surface, thereby resulting in much larger cross sections even at high energies. They increase in the low energy region, particularly for low values of $v$, up to a maximum where they start to decrease, and vibrational excitation of the target atom is effective, as expected, in promoting the reaction in the low energy region but it becomes an inhibiting factor as soon as 
Fig. 16 Left panel quantum ER cross sections for states (black, red, green and blue lines for $v=0,1,2$ and 3 , respectively) in the chemisorption well. Right panel quantum CID cross sections (lines as in the left panel) and ER cross sections for the indicated values of $v$ over an extended energy region. Vertical bars mark the binding energies for the initial target states considered

Fig. 17 Average internal energy (left), and vibrational (middle) and rotational (right) quantum numbers of the product $\mathrm{H}_{2}$ molecules. Line as in Fig. 16 different target vibrational
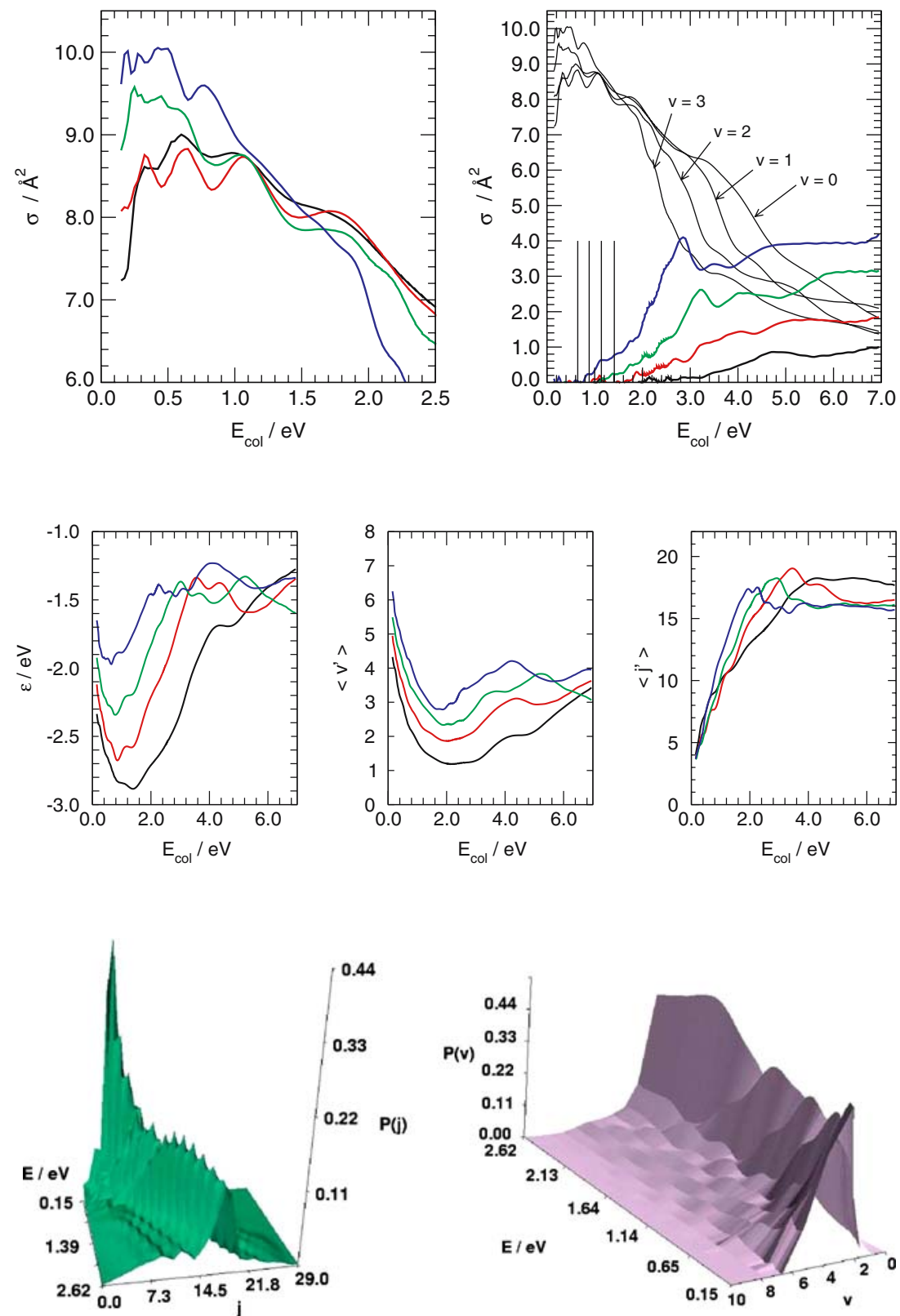

Fig. 18 Rotational and vibrational distributions for $v=0$ as functions of the incidence energy. Left panel vibrationally summed, rotational distributions. Right panel rotationally summed, vibrational distributions tions steeply point to low-lying vibrational states (Fig. 18), and show a particular structure suggesting that vibrational levels are selectively populated to some degree. This is indeed the case at least for target atoms initially in the $v=0$ state, for which the oscillations in the total reaction cross sections may be neatly assigned to selective population of the low-lying vibrational states of the product molecule, see Fig. 19 where the vibrationally resolved cross sections for $v^{\prime}=0-4$ are reported (multiplied by a factor of 2) along with the total reaction cross section shown in Fig. 16. A detailed analysis aimed to understand the origin of this behavior was performed with classical (CT) and quasi-classical (QCT) means [26]. This is justified since classical mechanics 


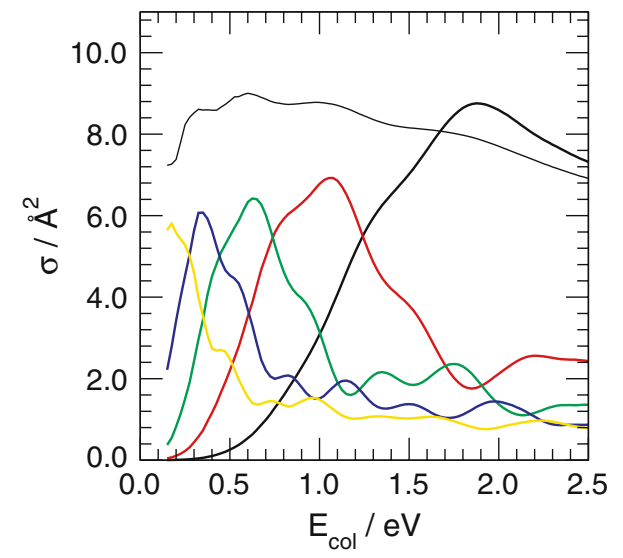

Fig. 19 Vibrationally resolved cross sections for a chemisorbed target atom initially in the $v=0$ state. Thin line represents the total ER cross sections of Fig. 16 and black, red, green, blue and yellow bold lines are the $v^{\prime}=0-4$ resolved cross sections (multiplied by 2)

reproduces well the average quantum results for the total reaction cross sections (see Fig. 20).

Briefly, it turned out that vibrational excitation of product molecules decreases, at a given energy, when increasing the impact parameter. This high impact parameter region gives the largest contribution to the cross section, and therefore high impact parameter trajectories are mainly responsible of the particular behavior of the overall vibrational excitation. For these trajectories (Fig. 21), the incident atom is only slightly deflected in its incoming motion, collides with the surface while the target gets nearer, and picks it at a distance close to the equilibrium value (for the resulting high $j$ value), thereby leading to molecules with very little vibrational excitation [the energy may be even less than the zero point value; this occurs in CT and QCT $(v=0)$ dynamics at about $2.0 \mathrm{eV}$. Classical vibrational distribution turned out to be in reasonable agreement with quantum results, but failed, of course, in reproducing the structure of Fig. 18. It seemed reasonable, therefore, to conclude [25,26] that classical mechanics is adequate in describing the dynamical behavior of the system but, in forming product molecules in low-lying, widely spaced vibrational levels, a quantum selection of the final state must be considered. This is the origin of the oscillations seen in Fig. 16, at least for collisions with the target initially in the $v=0$ state.

Analogously, the peak structure in the CID cross section was attributed to population of quasi-quantized metastable states of rotationally excited transient hydrogen molecules. Indeed, ER and CID were found to be, in this case, really competitive processes. "Transition" from ER reaction to CID occurs through formation of intermediate, metastable hydrogen molecules, that is CID partly follows a two-step mechanism, hydrogen formation and dissociation. Also in this case, classical mechanics was found to be in agreement with quantum mechanics but gave a much less pronounced structure in the cross sections (Fig. 22).
Fig. 20 QCT and quantum Eley-Rideal total cross sections for $v=0-3$ (black, red, green and blue, respectively) in the chemisorbed well. Bold lines for quantum results, points connected with lines for QCT results (bars represent estimated standard deviations). Dotted line in the lower left panel is for CT results
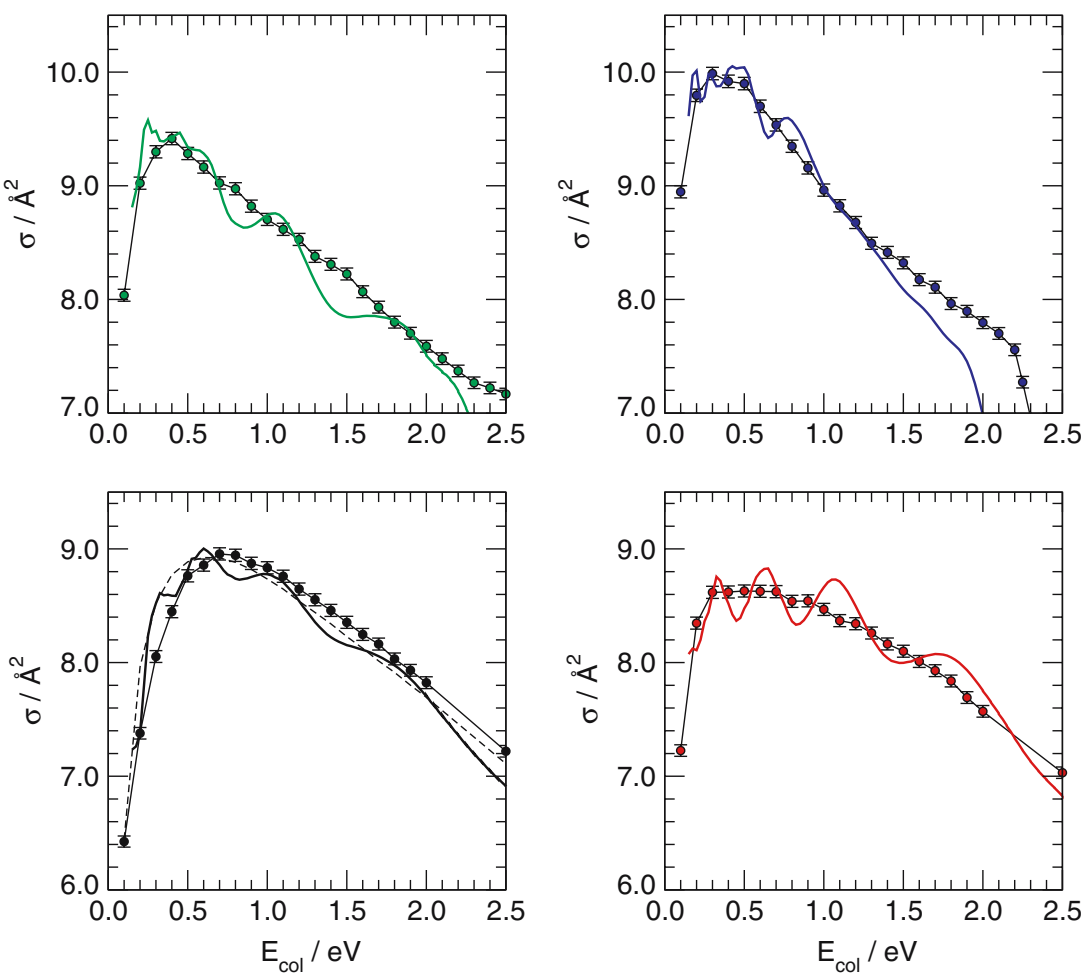


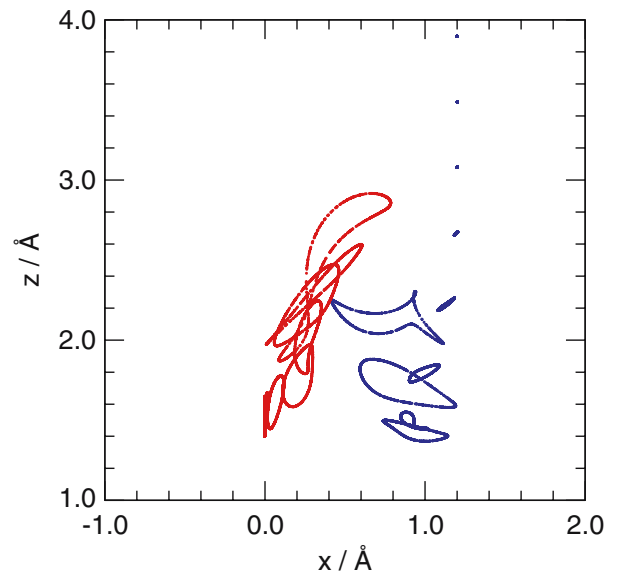

Fig. 21 "Snapshots" of a QCT $(v=0)$ bunch of trajectories at $E=1.5 \mathrm{eV}$ and $b=1.2 \AA$, showing the position of incident (blue) and chemisorbed target (red) atoms

\subsection{Adsorbate-induced trapping}

Eley-Rideal and CID results of the quantum calculations for target hydrogen atoms initially physisorbed on the surface are reported in Fig. 23, left and right panel, respectively. ER cross sections at low energies are large, somewhat larger than in the case in which the target atom was chemisorbed on the surface Fig. 16. This is due to the very weak attraction experienced by hydrogen atoms on the surface which allows on the one hand for target atoms being quite far from the surface and on the other hand for (low energy) projectiles to be steered toward the target by the strong $\mathrm{HH}$ attraction. Vibrational excitation of the target atom strongly inhibits hydrogen formation, because of the presence of the competing CID process. CID channel readily opens at the energetic thresholds and rapidly reaches a large saturation value, $\sigma \geq 10 \AA^{2}$. ER reaction products are vibrationally very hot, much hotter than before, and QCT results are in good agreement with quantum ones, except for a fine structure.

The interesting result, in this "physisorbed case", was the occurrence of a sizable trapping process. In general, among CI processes, trapping of the incident atoms due to scattering with adsorbates may be important for a correct understanding of surface recombination.
Fig. 22 Collision induced desorption cross sections for $v=0-3$ (black, red, green and blue, respectively) in the chemisorbed well. The quantum cross sections (solid lines) of Fig. 16 and the QCT results, with (points connected by bold lines) and without (points connected by thin lines) the contribution of metastable hydrogen molecules
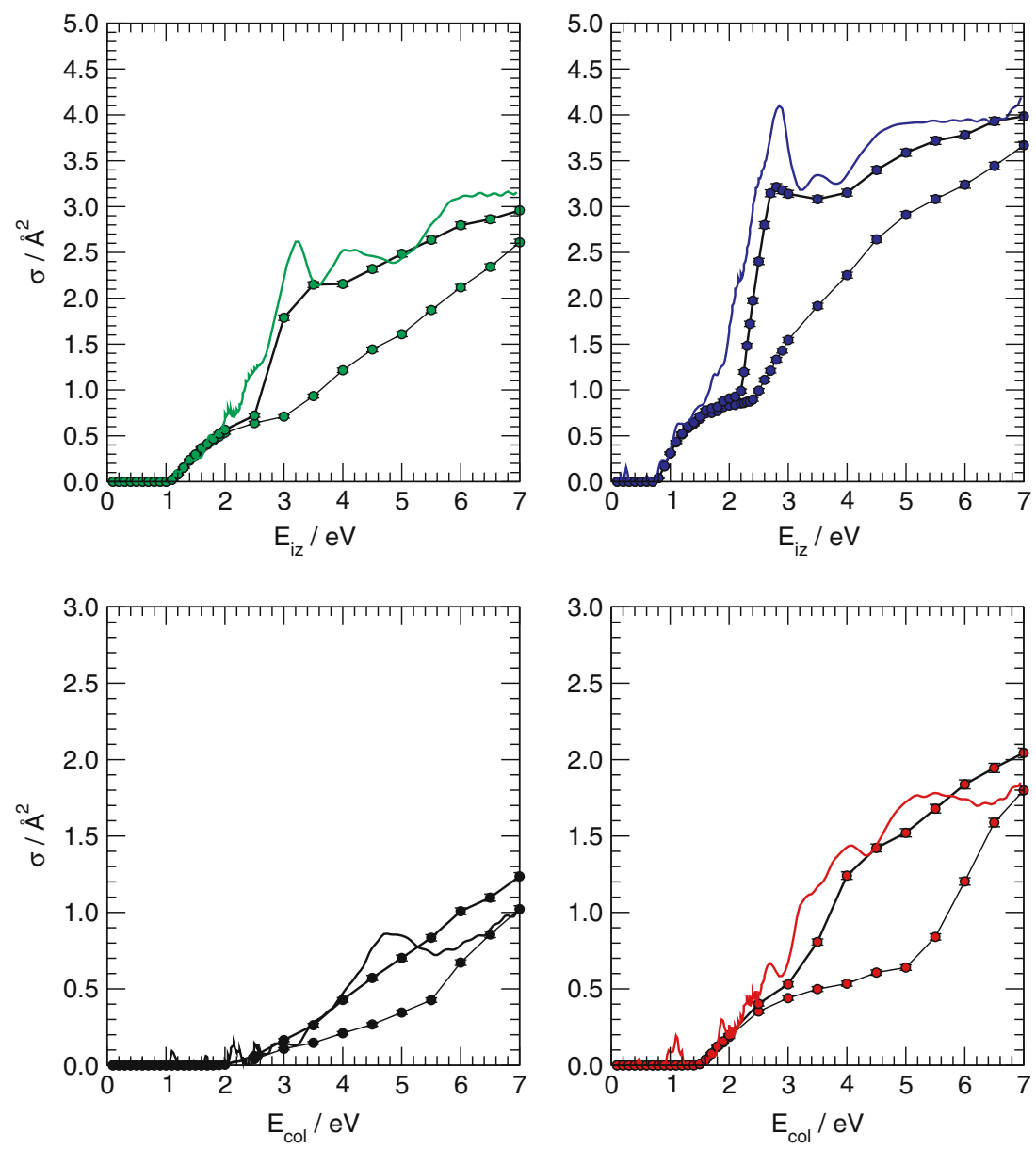
Fig. 23 ER(left panel) and CID (right panel) cross sections for the physisorbed case. Black, red, green and blue lines for the target vibrational quantum numbers $v=0-3$, respectively

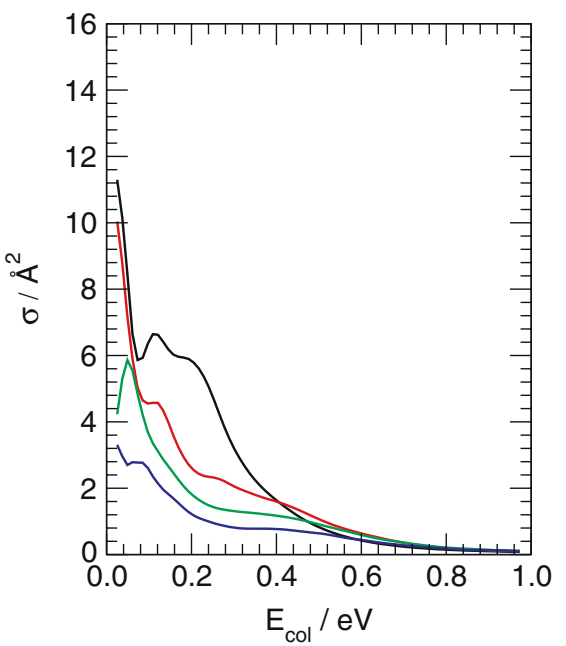

This is particularly true for light atoms scattering off weakly corrugated surfaces. Indeed, the absence of surface corrugation and of a favorable mass ratio between gas-phase and substrate atoms prevent energy conversion (diffraction) and energy exchange with the substrate (phonon excitation). Thus, the more favorable mass ratio with an adsorbate and the adsorbate induced local corrugation are factors which make the adsorbatemediated trapping an important process. Trapping may result either in formation of two species bound to the surface $(\mathrm{A}+\mathrm{BS} \rightarrow \mathrm{AS}+\mathrm{BS})$ or in an exchange process $(\mathrm{A}+\mathrm{BS} \rightarrow \mathrm{AS}+\mathrm{B})$ and is expected to produce hot atoms. As already mentioned in Sect. 3, these hot atoms travel for several angstroms across the surface and, depending on their energy contents and on the adsorbate-substrate dynamical features, they may relax after dissipating some energy to the substrate or may desorb after scattering off a lattice site. In the rigid, flat surface study considered in our study, both energy dissipation and surface corrugation were neglected and, thus, only the rate of hot atom formation could be computed.

Results showed that the efficiency of the trapping process was comparable to that of the ER reaction at low energies and considerable higher at high energies, where the cross sections roughly level off (Fig. 24). A more detailed analysis revealed that at these high energies, say $E \geq 0.2 \mathrm{eV}$, trapping occurs mainly because of the exchange process, whereas at low energy results in formation of two species bound to the surface.

A comparison with the quasi-classical trajectory results revealed that also in this case a classical picture is adequate, except for the structure observed in the quantum cross sections. This structure seems to be due to the contribution of the quasi-collinear approach of the projectile to the target (Fig. 25), thereby suggesting some strict relationship with the target vibrational motion. However, a deeper analysis was beyond the scope of the work. More importantly, it is worth noting that for trapping of the incident atom to occur is only necessary that the residual energy in the motion normal to the surface is less than the H-graphite binding energy. Indeed, analysis of the energy contents of the trapped atoms revealed that they have a total energy well above the desorption threshold, but this is mainly channeled in the motion parallel to the surface, see Fig. 24 where the empty symbols are the results for trapped projectile atoms with total energy less than the binding energy to the surface. This behavior is similar to that found for $\mathrm{H}$ on the $\mathrm{Ni}(100)$, Sect. 3, where cross sections of about $2 \AA^{2}$ were found for the (adsorbate-induced) formation of metastable hot atoms. The difference is that in that case a consistent fraction of bound hot atoms formed, too, thanks to the much higher binding energy of $\mathrm{H}$ on metals.

The importance of these trapped, high mobile species in the context of hydrogen formation in ISM has yet to be established. We note only that, in general, consideration of CID and adsorbate-induced trapping are expected to be important for a correct modeling of the reaction processes. CID of weakly bound species might help to "refresh" the surface and set free physisorbed sites useful for recombination. And, on the other hand, the presence of physisorbed hydrogen molecules (either coming from the gas-phase or produced by a surface reaction) and eventually of other atoms (e.g. He), might also help $\mathrm{H}$ atoms to become trapped and mobile on the surface. This could be important, since as already mentioned above, unfavorable adsorbate-substrate mass-ratio and small surface corrugation prevent energy dissipation and diffraction, and make sticking hard unless the collision energy is very low. 
Fig. 24 Comparison between QCT (filled symbols) and quantum (solid lines) for the total trapping cross sections of the incident atom. Empty symbols represent trapped atoms with an energy less than the desorption threshold (from QCT calculations). Black, red, green and blue for $v=0-3$ in the physisorbed well, respectively
Fig. 25 A representative set of classical trajectories in the $\rho, z_{i}$ plane, showing trajectories ending in the collision induced desorption channel (blue lines) and in trapping of the incident atom (red lines)
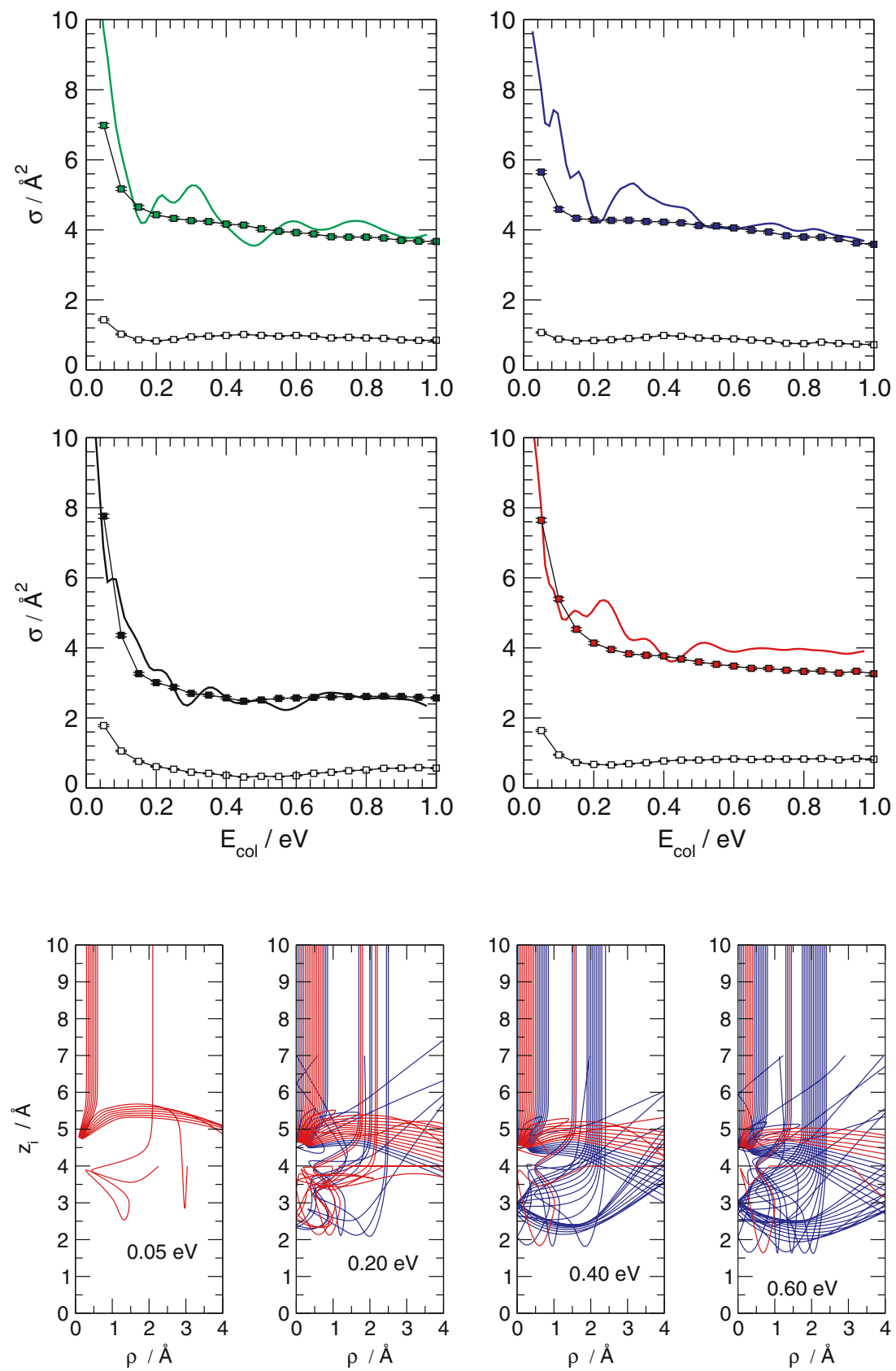

Structure, energetics and dynamics have been becoming ever and ever entangled as detailed informations come from new experiments. The objective would be, therefore, using first-principles calculations to sample that part of the energy landscape which is relevant for the process in consideration. Within a classical framework, an ab initio molecular dynamics (AIMD) approach would be of great value, e.g. to understand oxygen dynamics on aluminum. The approach is very appealing since it avoids the (human) time consuming step of producing an analytical representation of first-principle data. In this context, however, one has 
always to keep in mind that with current computational resources good sampling of initial conditions cannot be achieved with AIMD, and therefore AIMD results need some cautions.

In other cases, the main interest is in understanding general principles governing dynamical processes. Thus, for example, in studying hydrogen relaxation and diffusion on metal surfaces a reliable model including lattice vibrations is more valuable than accurate, but limited, first-principles informations. Here, two main problems are worth considering. The first is the role of non-adiabatic effects, i.e. electron-hole excitations, since interaction with metals always makes suspect the validity of the (fundamental) Born-Oppenheimer approximation. Despite in some cases combined experimental and theoretical efforts allowed to rule out such non-adiabatic effects in hydrogen scattering on a metal surface [5], there is evidence that in other systems they do play a role [156]. The second, important problem is the role of quantum mechanics in light atom dynamics. Classical dynamics may provide a good description of system dynamics even in the presence of hydrogen atoms if (e.g. in Eley-Rideal reactions on metal surfaces) strong, attractive forces accelerate light atoms. However, it may seriously fail if one considers slowly moving hydrogen atoms and/or low temperature surfaces.

This is surely the case, for example, of hydrogen dynamics on graphite, where the astrophysical interest asks for reactions of gas-phase atoms with surfaces both at very low temperature. Diffusion of hydrogen atoms on graphite might occur in the physisorbed state even at very low temperature thanks to quantum tunneling, and it is worth considering in order to assess the role of the Langmuir-Hinshelwood pathway for hydrogen formation. Here, even the zero-temperature diffusion coefficient (i.e. the ground-state tunneling contribution to diffusion) requires accurate informations on the energy landscape, which cannot be obtained by DFT methods because of current limitation with weak, van der Waals interactions. Furthermore, basic to hydrogen dynamics on graphite is the simple sticking problem which, in conditions relevant for the ISM, may become very challenging. Cold projectile atoms impinging on very cold surfaces require proper consideration of energy dissipation in a quantum mechanical framework. This is a typical system-bath quantum dynamical problem, for which the most elegant approach is time evolving the subsystem density matrix according to a Liouville-von Neumann equation including dissipation. Despite tremendous progresses in the field of open system dynamics, important problems remain in deriving a reliable, yet manageable, master equation. In light of these difficulties, we have recently introduced an alternative method
$[157,158]$ which considers the quantum evolution of the whole (subsystem + bath) system state in an approximate way. Comparison of our results with those of exact multi-configuration time-dependent Hartree [159] calculations in model systems with hundreds bath degrees of freedom suggests that the novel method is quite accurate and can be used in realistic, surface science problems.

\section{References}

1. Besenbacher F, Chorkendorff I, Clausen BS, Hammer B, Molenbroek AM, Nørskov JK, Stensgaard I (1998) Science 279:1913

2. Jacobsen CJH, Dahl S, Boisen A, Clausen BS, Topsøe H, Logadottir A, Nørskov JK (2002) J Catal 205:382

3. Honkala K, Hellman A, Remediakis IN, Logadottir A, Carlsson A, Dahl S, Christensen CH, Nørskov JK (2005) Science 307:555

4. Wodtke AM (2006) Science 312:64

5. Nieto P, Pijper E, Barredo D, Laurent G, Olsen RA, Baerends E-J, Kroes G-J, Farias D (2006) Science 312:86

6. Tully JC (2006) Science 312:1004

7. Liu Z, Feldman LC, Tolk NH, Zhang Z, Cohen PI (2006) Science 312:1024

8. Somorjai GA (1994) Introduction to surface chemistry and catalysis Wiley, New York

9. Groß A (2002) Surf Sci 500:347

10. Stampfl C, Ganduglia-Pirovano MV, Reuter K, Scheffler M (2002) Surf Sci 500:368

11. Rosei F, Rosei R (2002) Surf Sci 500:395

12. Bonn M, Kleyn AW, Kroes G-J (2002) Surf Sci 500:475

13. Kasemo B (2002) Surf Sci 500:656

14. Rod TH, Nørskov JK (2002) Surf Sci 500:678

15. Kolb DM (2002) Surf Sci 500:722

16. Zaera F (2002) Surf Sci 500:947

17. Groß A (2002) Theoretical surface Science: a microscopic Perspective. Springer, Berlin Heidelberg New York

18. Feibelman PJ (2002) Science 295:99

19. Held G, Menzel D (1994) Surf Sci 316:92

20. Truong TN, Truhlar DG, Garrett BC (1989) J Phys Chem 1989:8227

21. Wonchoba SE, Hu W-P, Truhlar DG (1995) Phys Rev B 51:9985

22. Martinazzo R, Assoni S, Marinoni G, Tantardini G (2004) J Chem Phys 120:8761

23. Sha X, Jackson B, Lemoine D (2002) J Chem Phys $116: 7158$

24. Sha X, Jackson B (2002) Surf Sci 496:318

25. Martinazzo R, Tantardini GF (2005) J Phys Chem A 109:9379

26. Martinazzo R, Tantardini GF (2006) J Chem Phys 124

27. Thiel PA, Paola RAD, Hoffmann FM (1984) J Chem Phys 80:5326

28. Su X, Lianos L, Shen YR, Somorjai G (1998) Phys Rev Lett 80:1533

29. Iedema MJ, Dresser MJ, Doering DL, Rowland JB, Hess WP, Tsekouras AA, Cowin JP (1998) J Phys Chem B 102:9203

30. Witek H, Buch V (1999) J Chem Phys 110:3168

31. Thiel PA, Madey TE (1987) Surf Sci Rep 7:211

32. Henderson MA (2002) Surf Sci Rep 46:1

33. Madey TE, Yates JT (1977) Chem Phys Lett 51:77

34. Doering DL, Madey TE (1982) Surf Sci 123:305 
35. Thiel PA, Hoffmann FM, Weinberg WH (1981) J Chem Phys 75:5556

36. Williams ED, Doering DL (1983) J Vac Sci Technol A 1:1188

37. Schmitz PJ, Polta JA, Chang SL, Thiel PA (1987) Surf Sci $186: 219$

38. Kretzschmar K, Sass JK, Bradshaw AM, Holloway S (1982) Surf Sci 115:183

39. Pirug G, Ritke C, Bonzel HP (1991) Surf Sci 241:289

40. Hobbs PV (1974) Ice physics Clarendon, Oxford

41. Bernal JD, Fowler RH (1933) J Chem Phys 1:515

42. Held G, Menzel D (1995) Surf Sci 327:301

43. Held G, Menzel D (1995) Phys Rev Lett 74:21

44. Menzel D (2002) Science 295:58

45. Michaelides A, Alavi A, King DA (2003) J Am Chem Soc 125:2746

46. Feibelman PJ (2003) Phys Rev B 67:35420

47. Denzler DN, Hess C, Dudek R, Wagner S, Frischkorn C, Wolf M, Ertl G (2003) Chem Phys Lett 376:618

48. Miranda PB, Xu L, Shen YR, Salmeron M (1998) Phys Rev Lett 81:5876

49. Kubota J, Wade A, Domen K, Kano SS (2002) Chem Phys Lett 362:476

50. Segall MD, Lindan P, Probert MJ, Pickard CJ, Hasnip PJ, Clark SJ, Payne MC (2002) J Phys Condens Matter 14:2717

51. Payne MC, Teter MP, Allan DC, Arias TA, Joannopoulos JD (1992) Rev Mod Phys 64:1045

52. Perdew JP, Wang Y (1992) Phys Rev B 45:13244

53. Bachelet GB, Schlüter (1982) M Phys Rev B 25:2103

54. Kleinman L (1980) Phys Rev B 21:2630

55. Vanderbilt D (1990) Phys Rev B 41:7892

56. Materzanini G, Tantardini GF, Lindan PJD, Saalfrank P (2005) Phys Rev B 71:155414

57. Meng S, Xu LF, Wang EG, Gao S (2002) Phys Rev Lett 89:176104

58. Ogasawara H, Brena B, Nordkund D, Nyberg M, Pelmenschikov A, Pettersson LGM, Nilsson A (2002) Phys Rev Lett 89:276102

59. Puisto SR, Lerotholi TJ, Held G, Menzel D (2003) Surf Rev Lett 10:487

60. Held G, Pfnur H, Menzel D (1992) Surf Sci 271:21

61. Hoffmann W, Benndorf C (1997) Surf Sci 377:681

62. Lilach Y, Romm L, Livneh T, Asscher M (2001) J Phys Chem B 105:2736

63. Andersson K, Nikitin A, Pettersson LGM, Nilsson A, Ogasawara H (2004) Phys Rev Lett 93:196101

64. Feibelman PJ (2005) Phys Rev B 113405

65. Faradzhev NS, Kostov KL, Feulner P, Madey TE, Menzel D (2005) Chem Phys Lett 415:165

66. Haq S, Clay C, Darling GR, Zimbitas G, Hodgson A (2006) Phys Rev B 73:115414

67. Noguera C (1996) Physics and chemistry at oxide surfaces. Cambridge University Press, Cambridge

68. Szklarska-Smialowska Z (1999) Corros Sci 41:174367

69. Armstrong RD, Braham VJ (1995) Corros Sci 37:148996

70. Zhukov V, Popova I, Yates JT (1999) Surf Sci 441:251

71. Rhodin TN, Ertl G (eds) (1979) The nature of the surface chemical bond. North-Holland, Amsterdam

72. Barth JV (2000) Surf Sci Rep 40:75

73. Lanzani G, Tantardini GF (2006) (in preparation)

74. Perdew J, Burke K, Ernzerhof M (1996) Phys Rev Lett 77:3865

75. Perdew J, Burke K, Ernzerhof M (1998) Phys Rev Lett 80:891

76. Blochl PE (1994) Phys Rev B 50:17953

77. Kresse G, Furthmuller J (1996) Comput Mater Sci 6:15

78. Kresse G, Furthmuller J (1996) Phys Rev B 54:11169

79. Kresse G, Joubert J (1999) Phys Rev B 59:1758
80. Kresse G, Hafner J (1994) J Phys Condens Matter 6:8245

81. Hammer B, Nørskov JK (2000) Adv Catalys 45:71

82. Mitchel R, Castaldi J, Allasia C, Jourdan C, Derrien J (1980) Surf Sci 95:309

83. Wyckoff RWG (1964) Crystal structures. vol. 2, 2nd edn Wiley, New York

84. Weinberg WH (1991) Dynamics of gas-surface interactions. In: Rettner CT, Ashfold MNR (eds) The Royal Society of Chemistry, London

85. Rettner CT (1992) Phys Rev Lett 69:383

86. Rettner CT, Auerbach DJ (1995) Phys Rev Lett 74:4551

87. Rettner CT (1994) J Chem Phys 101:1529

88. Harris J, Kasemo B (1981) Surf Sci 105:L281

89. Jackson B, Persson M (1992) J Chem Phys 96:2378

90. Jackson B, Persson M (1995) J Chem Phys 103:6257

91. Persson M, Jackson B (1995) J Chem Phys 102:1078

92. Kammler T, Wehner S, Küppers J (1995) Surf Sci 339:125

93. Kammler T, Lee J, Küppers J (1997) J Chem Phys 106:7362

94. Zecho T, Brander B, Küppers J (1998) Surf Sci Lett 418: 418

95. Wehner S, Küppers J (1998) Chem Phys Lett 288:873

96. Wehner S, Küppers J (1998) J Chem Phys 109:294

97. Wehner S, Küppers J (1998) Surf Sci 411:46

98. Wehner S, Küppers J (1998) J Chem Phys 108:3353

99. Buntin SA (1997) Chem Phys Lett 278:71

100. Buntin SA (1998) J Chem Phys 108:1601

101. Kim J-Y, Lee J (1999) Phys Rev Lett 82:1325

102. Kim J-Y, Lee J (2000) J Chem Phys 113:2856

103. Tantardini GF, Simonetta M (1981) Surf Sci 105:577

104. Pazzi VI, Philipsen PHT, Baerends EJ, Tantardini GF (1999) Surf Sci 443:1

105. Pineau N, Busnengo HF, Rayez JC, Salin A (2005) J Chem Phys 122:214705

106. Rendulic KD, Anger G, Winkler A (1989) Surf Sci 208:404

107. Kresse G (2000) Phys Rev B 62:8295

108. Kresse G, Hafner J (2000) Surf Sci 459:287

109. Pino I, Martinazzo R, Tantardini GF (2006) (in preparation)

110. Meregalli V, Parrinello M (2001) Appl Phys A: Mater Sci Process 72:143

111. Mayer M, Philipps V, Wienhold P, Seggern HHJ, Rubel M (2001) J Nucl Mater 290-293:381

112. Ma Y, Xia Y, Zhao M, Ying M, Liu X, Liu P (2001) J Chem Phys 115:8152

113. Ma Y, Xia Y, Zhao M, Wang R, Mei L (2001) Phys Rev B 63:115422

114. Gould RJ, Salpeter EE (1963) Astrophys J 138:393

115. Hollenbach DJ, Salpeter EE (1970) J Chem Phys 53:79

116. Hollenbach DJ, Salpeter EE (1971) Astroph J 163:155

117. Greenberg JM (2000) Surf Sci 500

118. Williams DA, Herbst E (2000) Surf Sci 500:823

119. Draine BT (2003) Ann Rev Astron Astrophys 41:241

120. Jeloiaca L, Sidis V (1999) Chem Phys Lett 300:157

121. Sha X, Jackson B (2002) Surf Sci 496:318

122. Ferro Y, Marinelli F, Allouche A (2002) J Chem Phys 116:8124

123. Allouche A, Ferro Y, Angot T, Thomas C, Layet JM (2005) J Chem Phys 123:124701

124. Zecho T, Guttler A, Kuppers J (2004) Carbon 42:609

125. Hornekær ZSL, Xu W, Otero R, Rauls E, Stensgaard I, Lægsgaard E, Hammer B, Besenbacher F (2006) Phys Rev Lett 96:156104

126. Hollenbach DJ, Tielens AGGM (1999) Rev Mod Phys 71:173

127. Ghio E, Mattera L, Salvo C, Tommasini F, Valbusa U (1980) J Chem Phys 73:556

128. Farebrother A, Meijer AJHM, Clary DC, Fisher AJ (2000) Chem Phys Lett 319:303 
129. Meijer AJHM, Farebrother A, Clary DC, Fisher AJ (2001) J Phys Chem A 105:2173

130. Jackson B, Lemoine D (2001) J Chem Phys 114:474

131. Sha X, Jackson B, Lemoine D (2002) J Chem Phys 116:7158

132. Morisset S, Aguillon F, Sizun M, Sidis V (2003) Phys Chem Chem Phys 5:506

133. Morisset S, Aguillon F, Sizun M, Sidis V (2003) Phys Chem Chem Phys 378:615

134. Morisset S, Aguillon F, Sizun M, Sidis V (2004) J Phys Chem A 108:8571

135. Morisset S, Aguillon F, Sizun M, Sidis V (2004) J Chem Phys 121:6493

136. Morisset S, Aguillon F, Sizun M, Sidis V (2005) J Chem Phys 122:194702

137. Zecho T, Guttler A, Sha X, Jackson B, Kuppers J (2002) J Chem Phys 117:8486

138. Sha X, Jackson B, Lemoine D, Lepetit B (2005) J Chem Phys 122:014709

139. Perry JSA, Price SD (2003) Astrophys Space Sci 285:769

140. Creighan SC, Perry JSA, Price SD (2006) J Chem Phys 124:114701

141. Pirronello V, Biham O, Liu C, Shen L, Vidali G (1997) Astrophys J 483:L131

142. Pirronello V, Liu C, Shen L, Vidali G (1999) Astrophys J 475:L69

143. Katz N, Furman I, Biham O, Pirronello V, Vidali G (1999) Astrophys J 522:305
144. Hornekaer L, Baurichter A, Petrunin V, Field D, Luntz A (2003) Science 302:1943

145. Asscher M, Romm L, Zeiri Y (2002) Colloids and surfaces 208:187

146. Asscher M, Zeiri Y (2003) J Phys Chem B 107:6903

147. Althorpe SC, Fernandez-Alonso F, Bean BD, Ayers JD, Pomerantz AE, Zare RN, Wrede E (2002) Nature 416:67

148. Harich SA, Dai D, Wang CC, Yang X, Chao SD, Skodje RT (2002) Nature 419:281

149. Dai D, Wang CC, Harich SA, Wang X, Yang X, Chao SD, Skodje RT (2003) Science 300:1730

150. Skodje RT, Yang X (2004) Int Rev Phys Chem 23:253

151. Skodje RT, Skouteris D, Manolopoulos DE, Lee SH, Dong F, Liu K (2000) Phys Rev Lett 85:1206

152. Skodje RT, Skouteris D, Manolopoulos DE, Lee SH, Dong F, Liu K (2000) J Chem Phys 112:4536

153. Shiu W, Lin JJ, Liu K (2004) Phys Rev Lett 92:103201

154. Zhou J, Lin JJ, Liu K (2004) J Chem Phys 121:813

155. Zhang B, Liu K (2005) J Chem Phys 122:101102

156. Luntz AC, Persson M, Sitz GO (2006) J Chem Phys 124:091101

157. Martinazzo R (2005) (unpublished)

158. Martinazzo R, Nest M, Saalfrank P, Tantardini GF (2006) J Chem Phys 125:194102

159. Beck MH, Jackle A, Worth GA, Meyer H-D (2000) Phys Rep 324:1 